\title{
LINEARIZATION OF COMPLEX HYPERBOLIC DULAC GERMS
}

\author{
D. PERAN ${ }^{1}$, M. RESMAN ${ }^{2}$, J.P. ROLIN ${ }^{3}$, T. SERVI ${ }^{4}$
}

\begin{abstract}
We prove that a hyperbolic Dulac germ with complex coefficients in its expansion is linearizable on a standard quadratic domain and that the linearizing coordinate is again a complex Dulac germ. The proof uses results about normal forms of hyperbolic transseries in [PRRS21].
\end{abstract}

\section{Contents}

1. Introduction

2. Notation and main results

2.1. Definitions and Notation

2.2. The main result: Theorem B

3. Analytic linearization

3.1. Admissible domains

3.2. Examples

3.3. Linearization theorem for holomorphic maps on spiraling domains: Theorem A

4. Linearization of Dulac maps

4.1. Linearization of a hyperbolic Dulac series

4.3. Proof of Theorem B: uniqueness and existence of a (complex) Dulac linearization

References

\section{INTRODUCTION}

Given the germ $f$ of a real or complex function in one variable at the fixed point 0 , the linearization of $f$ consists in finding a number $\lambda$ and a change of coordinates $\varphi$ which solves Schröder's equation $\varphi(f(z))=\lambda \varphi(z)$ [Sch70]. In the differentiable case, $\lambda$ is the multiplier $f^{\prime}(0)$. A goal of the linearization is to embed $f$ in a flow, which allows to define the so-called "fractional iterates" of $f$ by the formula $f^{[t]}(z):=\varphi^{-1}\left(\lambda^{t} \varphi(z)\right)$ for $t \in \mathbb{R}$ (or $t \in \mathbb{C}$ ). Schröder's equation has been solved

2010 Mathematics Subject Classification. 34C20, 37C25, 39B12, 47H10, 12 J15.

Key words and phrases. Dulac germs and series, hyperbolic fixed point, linearization, Koenigs' sequence.

This research of D. Peran and M. Resman is partially supported by the Croatian Science Foundation (HRZZ) grant UIP-2017-05-1020. The research of M. Resman is also partially supported by the Croatian Science Foundation (HRZZ) grant PZS-2019-02-3055 from Research Cooperability funded by the European Social Fund. The research of all four authors is partially supported by the Hubert-Curien 'Cogito' grant 2021/2022 Fractal and transserial approach to differential equations. 
by Koenigs in the case of a holomorphic germ $f$ at $0 \in \mathbb{C}$ with a hyperbolic attractive fixed point (that is, when $0<|\lambda|<1$ ) [Koe84, CG93, Mil06]. Koenigs' method consists in proving that the so-called Koenigs sequence $\left(\varphi_{n}\right)$, defined by $\varphi_{n}=\frac{1}{\lambda^{n}} f^{\circ n}$, converges uniformly to a holomorphic solution $\varphi$ called the Koenigs linearizing coordinate. Moreover, $\varphi$ is a biholomorphism which is tangent to the identity, that is $\varphi(z)=z+o(z)$.

The problem of the convergence of Koenigs' sequence for more general germs has been tackled by various authors. Kneser proved the convergence of the Koenigs sequence for a hyperbolic attracting real germ $f$ such that $f(x)=\lambda x+O\left(|x|^{1+\delta}\right)$ for $x \rightarrow 0$, for some $\delta>0$ [Kne49]. However, this result does not help much for the purposes of iteration since, under Kneser's hypotheses, the limit $\varphi$ of Koenigs' sequence may not admit a compositional inverse (so that $\varphi$ is not, strictly speaking, a change of coordinates). To overcome this problem, Szekeres imposed stronger conditions on $f$ [Sze58]. He proved that if $f$ is continuous and has a differentiable representative on an interval $(0, d)$ which is strictly increasing, with $0<f(x)<x$ on $(0, d)$, and if $f^{\prime}(x)=\lambda+O\left(x^{\delta}\right)$ for $x \rightarrow 0$, for some $0<\lambda<1$ and $\delta>0$, then its Koenigs sequence converges on $(0, d)$ to a differentiable and strictly increasing solution. A simpler proof for real germs of class $\mathcal{C}^{r}, r \geq 2$, has been provided by Sternberg [Ste57, Nav11].

More recently, the convergence of Koenigs' sequences for maps admitting an asymptotic behavior in the scale of iterated logarithms has been considered [DF03]. More precisely, if $f$ is an interval map of class $\mathcal{C}^{1}$ defined in a neighborhood of the fixed point 0 with $f^{\prime}(0)=\lambda, 0<\lambda<1$, such that

$$
f(x)=\lambda x+O\left(\frac{x}{y \log (y) \cdots \log ^{\circ(p-1)}(y)\left(\log ^{\circ p}(y)\right)^{1+\varepsilon}}\right)
$$

for $x \rightarrow 0$, for some $\varepsilon>0$ and a nonnegative integer $p$, where $y:=-\log (|x|)$, then the Koenigs sequence of $f$ converges uniformly on a neighborhood of 0 to a limit $\varphi$ such that $\varphi(0)=0$ and $\varphi^{\prime}(0)=1$. The exponent $\varepsilon>0$ plays an important role here, as shown by the following example from [Ste57]. If $f$ is defined by $f(x)=x\left(\lambda-\frac{1}{\log (x)}\right)$ for $x \in(0, d], d>0$, and $f(0):=0$, then its Koenigs sequence diverges on $(0, d]$ (see also [DF03, Nav11]).

The previous result on functions with a logarithmic asymptotic behavior leads us naturally to a class of maps studied increasingly in the last few years, namely, maps which admit a transserial asymptotic expansion at 0 . In a word, a transseries is a generalized series whose monomials involve the exponential and logarithm functions. Maps (or germs of maps) which admit at their fixed point a transseries as their asymptotic expansion are studied by physicists nowadays (see for example [ABS19]). They also appear in dynamical systems and differential equations, e.g. as first return maps of polycycles of polynomial vector fields, studied in detail by Écalle [Éca92] and Il'yashenko [Il'91] in their respective proofs of Dulac's Conjecture. In this case, while being generated by a continuous dynamical system (a polynomial vector field), these maps are viewed as discrete dynamical systems on 
the real line. It is hence relevant to study them from the point of view of iteration theory. Their properties reveal the features of the generating system. In particular, the multiplicity of such a map in a parametric family is linked to the cyclicity of the polycycle [Rou98]. In this spirit, the orbits of some of them have been recently analyzed from the point of view of "fractal analysis" to read the cyclicity in bifurcations or formal normal forms [ZZ08, MRŽ12, R13].

Among these first return maps, some are of particular importance. In this paper, we consider Dulac maps, which are called almost regular germs by Il'yashenko [II'84]. The first return maps of nondegenerate polycycles of saddle type belong to the class of Dulac germs. They are analytic on an open interval $(0, d)$, and their (trans)asymptotic expansion at 0 is a (possibly) infinite sum of powers of the variable multiplied by real polynomials in the logarithm of the variable (what Il'yashenko calls in [Il'84] a Dulac series). Moreover, a remarkable achievement of Il'yashenko is the following quasianalyticity result: a Dulac map is equal to the identity if and only if its asymptotic expansion is equal to the identity. In order to prove this, he showed (considering complexifications in $\mathbb{C}^{2}$ of planar saddles) that Dulac maps can be analytically extended to sufficiently big complex domains (called standard quadratic domains) of the Riemann surface of the logarithm. Then, due to the fact that these extensions decay faster than exponentially at infinity on these domains, the conclusion follows by a version of the maximum modulus principle, the Phragmen-Lindelöf theorem [Il'84].

The Dulac maps play a crucial role in Il'yashenko's study of Dulac's Conjecture about non-accumulation of limit cycles on elementary polycycles, and have been extensively studied since. In particular, we are interested here in their normal forms and their embeddings in flows of vector fields, that is, in their study from the point of view of iteration theory.

This approach has been initiated in [MRRŽ19] and continued in [MR21]. There, the authors consider Dulac maps tangent to the identity, that is, with asymptotic expansions of the form $z+o(z)$, when $z \rightarrow 0$. It was proved that such a Dulac map can be conjugated on attracting and repelling sectors for its local dynamics, via a (sectorially) analytic change of coordinate called a Fatou coordinate, to the translation $t \mapsto t+1$. This sectorial Fatou coordinate admits a transserial asymptotic expansion, which is, however, more complicated than a Dulac series.

Our main result here is the linearization of complex hyperbolic Dulac germs (Theorem B). These are Dulac maps defined on standard quadratic domains whose asymptotic Dulac series, with possibly complex coefficients, are of the form $\lambda z+o(z)$, for $\lambda \in \mathbb{C}, 0<|\lambda|<1$, uniformly in the domain as $|z| \rightarrow 0$. We prove that a complex Dulac map admits on a standard quadratic domain a linearizing Koenigs coordinate, which is itself a complex Dulac map tangent to the identity (Theorem B in Section 2). Although the Dulac germs appearing as first return maps around saddle type polycycles of planar vector fields have only real coefficients in the expansion, the interest of complex Dulac germs lies in the fact that they appear as corner maps of hyperbolic complex saddles in $\mathbb{C}^{2}$, see e.g. Section 7 in [Lor05]. 
Two main tools are used in the proof. First, we prove a general result, which is an extension of Koenigs' and Dewsnap-Fisher's results: if $f(z)=\lambda z+o(z)$ when $|z| \rightarrow 0$, and $0<|\lambda|<1$, is a hyperbolic analytic map on a convenient invariant subdomain of the Riemann surface of the logarithm, and if it has on this domain an asymptotic behavior similar to the one considered in [DF03], then the Koenigs sequence of $f$ converges on the same subdomain to an analytic linearizing map $\varphi$ tangent to the identity (Theorem A in Section 3). Note that we do not request in Theorem A any particular asymptotic behavior after the first term. Secondly, using the results of [PRRS21] on the linearization of hyperbolic logarithmic transseries, we prove that, if $f$ is a hyperbolic Dulac map on a quadratic domain, then its Koenigs coordinate is also a Dulac map (see Section 4). This last fact marks a difference with the parabolic case studied in [MRRŽ19], where it was proven that the normalizing coordinate of a parabolic Dulac map is in general not a Dulac map, but belongs to some bigger class of germs with logarithmic transserial asymptotic expansions.

Our theorems are thus a generalization of the standard Koenigs linearization result for hyperbolic analytic germs from $\operatorname{Diff}(\mathbb{C}, 0)$ to hyperbolic germs which are not necessarily analytic at a fixed point, on their invariant subdomains of the Riemann surface of the logarithm.

\section{NOtATION AND MAIN RESUlts}

2.1. Definitions and Notation. In what follows, $z$ denotes a complex infinitesimal variable.

We first give the definition of a complex Dulac series and of a complex Dulac germ. We are motivated by the definition of Dulac series and of almost regular germs given in [Il'91]. However, while the definitions there were adapted to the Dulac problem for real vector fields, we work here with series with complex coefficients.

In order to define an almost regular germ, we recall a few classical definitions. We denote by $\widetilde{\mathbb{C}}:=\left\{(r, \theta): r \in \mathbb{R}_{>0}, \theta \in \mathbb{R}\right\}$ the Riemann surface of the logarithm. By a classical abuse of notation, we write its elements $(r, \theta)$ as $z=r \mathrm{e}^{\mathrm{i} \theta}$, where $r=|z|>0$ and $\theta=\arg (z) \in \mathbb{R}$. A (spiraling) neighborhood of the origin in $\widetilde{\mathbb{C}}$ is a set of the form $\mathcal{V}=\left\{r \mathrm{e}^{\mathrm{i} \theta}: 0<r<h(\theta)\right\}$, where $h: \mathbb{R} \rightarrow(0,+\infty)$ is a continuous function. Two functions define the same germ at the origin of $\widetilde{\mathbb{C}}$ if they coincide on some spiraling neighborhood of the origin in $\widetilde{\mathbb{C}}$.

We endow $\widetilde{\mathbb{C}}$ with a structure of one-dimensional analytic Riemann manifold whose atlas consists of a single chart, called the logarithmic chart,

$$
-\log : \widetilde{\mathbb{C}} \rightarrow \mathbb{C}, z=r \mathrm{e}^{\mathrm{i} \theta} \mapsto \zeta=-\log z=-\log r-\mathrm{i} \theta .
$$

Hence $z=\exp (-\zeta)=\mathrm{e}^{-\zeta}$. Notice that $z \rightarrow 0$ (in the sense that $|z| \rightarrow 0$ ) when $\zeta \rightarrow \infty$ on $\mathbb{C}$ (in the sense that $\Re(\zeta) \rightarrow+\infty$ ).

A function $\widetilde{f}: \widetilde{A} \subseteq \widetilde{\mathbb{C}} \rightarrow \widetilde{\mathbb{C}}$ is called analytic if its representation $f: \zeta \mapsto$ $-\log \left(\widetilde{f}\left(\mathrm{e}^{-\zeta}\right)\right)$ on the domain $A=-\log (\widetilde{A})$ of the logarithmic chart $\mathbb{C}$ is analytic. In what follows, we often switch for convenience between the presentation of a 
domain or a function in the $z$-chart and its presentation in the logarithmic chart $\zeta=-\log z$ (also called the $\zeta$-chart).

Finally, let $\mathbb{C}^{+}=\{\zeta \in \mathbb{C}: \Re(\zeta)>0\}$.

Following [IY08, Section 24], given $C>0$, the standard quadratic domain $\mathcal{R}_{C} \subset$ $\mathbb{C}$ is the set defined in the logarithmic chart $\mathbb{C}$ as

$$
\kappa\left(\mathbb{C}^{+}\right) \text {, where } \kappa(\zeta)=\zeta+C(\zeta+1)^{\frac{1}{2}},
$$

(see Figure 1). The set $\widetilde{\mathcal{R}}_{C} \subset \widetilde{\mathbb{C}}$ represented by $\mathcal{R}_{C}$ is a spiraling neighborhood of the origin in $\widetilde{\mathbb{C}}$.

Definition 2.1 (The germ of a standard quadratic domain $\mathcal{R}_{C}$ ). For $C>0$, let $\mathcal{R}_{C} \subset \mathbb{C}$ be the standard quadratic domain given by (2.1) in the $\zeta$-chart. We call the elements of the collection of sets

$$
\left\{\left(\mathcal{R}_{C}\right)_{R}=\mathcal{R}_{C} \cap([R,+\infty) \times \mathbb{R}): R>0\right\},
$$

the germs of the standard quadratic domain $\mathcal{R}_{C}$.

Remark 2.2. Let $C>0$. For each germ $\left(\mathcal{R}_{C}\right)_{R}$ of the standard quadratic domain $\mathcal{R}_{C}$, there exists sufficiently big $C_{0}>R$ such that for every $C^{\prime}>C_{0}$, standard quadratic domain $\mathcal{R}_{C^{\prime}}$ is entirely contained in $\left(\mathcal{R}_{C}\right)_{R}$. Indeed, for $C^{\prime}>C_{0}$, where $C_{0}$ is sufficiently big, it can be seen that $\mathcal{R}_{C^{\prime}} \subset \mathcal{R}_{C}$. Also, for every $\zeta \in \mathcal{R}_{C^{\prime}}$, it holds that $\Re(\zeta)>C^{\prime}>C_{0}>R$.

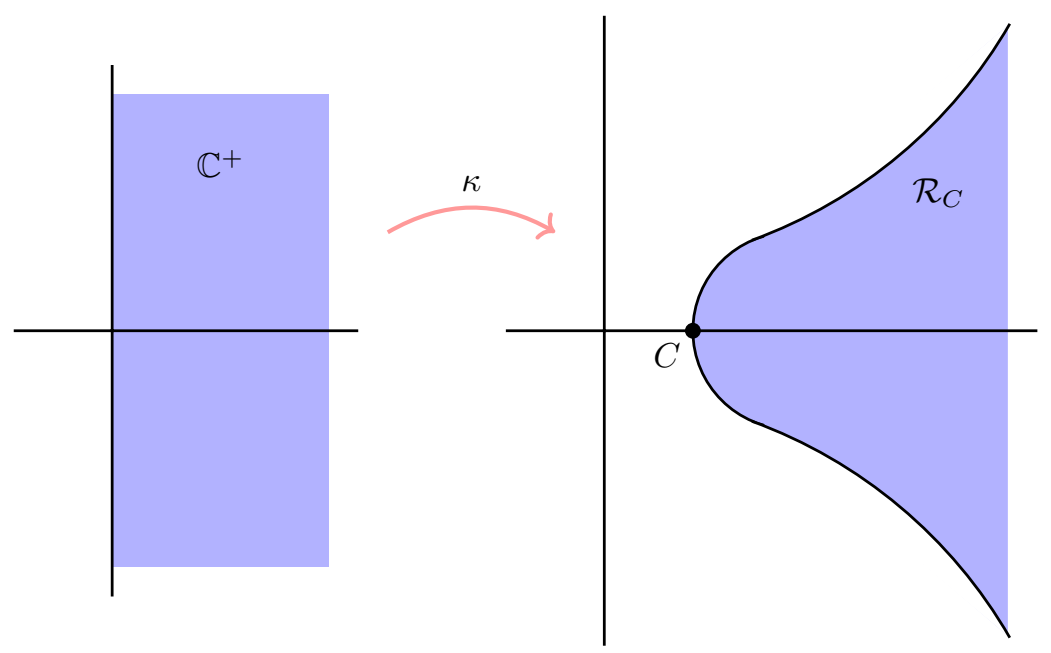

FIgURE 1 . The image of standard quadratic domain $\mathcal{R}_{C}$, for some $C>0$, in the $\zeta$-chart.

In analogy with [IY08, Section 24], a complex Dulac series is a transseries of the form

$$
\widehat{f}=\alpha \zeta+\beta+\sum_{i=1}^{\infty} P_{i}(\zeta) \exp \left(-\alpha_{i} \zeta\right), \quad \alpha \in \mathbb{R}_{>0}, \beta \in \mathbb{C}, P_{i} \in \mathbb{C}[\zeta]
$$

where $\left(\alpha_{i}\right)_{i \geq 1}$ is a strictly increasing sequence of positive real numbers belonging to a finitely generated sub-semigroup of $\left(\mathbb{R}_{>0},+\right)$, such that $\left(\alpha_{i}\right)_{i} \rightarrow+\infty$. 
The series $\widehat{f}$ is called parabolic if $\alpha=1$ and $\beta=0$, and is called hyperbolic if $\alpha=1$ and $\Re(\beta) \neq 0$.

A complex Dulac germ is a holomorphic germ $f$ on a standard quadratic domain $\mathcal{R}_{C}$, which admits on $\mathcal{R}_{C}$ an asymptotic expansion given by a complex Dulac series (2.2), uniformly on $\mathcal{R}_{C}$ in the following sense: for every $\nu>0$, there exists $N_{\nu} \in \mathbb{N}$, such that

$$
\left.\left|f(\zeta)-\alpha \zeta-\beta-\sum_{i=1}^{N_{\nu}} P_{i}(\zeta) \exp \left(-\alpha_{i} \zeta\right)\right|=o(\exp (-\nu \zeta))\right)
$$

as $\Re(\zeta) \rightarrow+\infty$ in $\mathcal{R}_{C}$.

Remark 2.3. A complex Dulac germ $f$ is represented in the $z$-chart by the germ $\tilde{f}$ which admits as $z \rightarrow 0$ an asymptotic expansion which is a logarithmic complex Dulac series, that is a transseries

$$
\lambda z^{\alpha}+\sum_{i=1}^{+\infty} z^{\beta_{i}} Q_{i}(-\log z), \quad \alpha>0, \lambda \in \widetilde{\mathbb{C}}, Q_{i} \in \mathbb{C}[X]
$$

where $\left(\beta_{i}\right)_{i \geq 1}$ is a strictly increasing sequence of real numbers strictly bigger than $\alpha$, belonging to a finitely generated sub-semigroup of $\left(\mathbb{R}_{>0},+\right)$, and which tends to $+\infty$.

If a complex Dulac series $\widehat{f}$ from (2.2) satisfies additionally $\beta \in \mathbb{R}_{\geq 0}$ and $P_{i} \in \mathbb{R}[\zeta]$ for all $i \geq 1$, then we call $\widehat{f}$ a real Dulac series. If a complex Dulac germ $f$ additionally satisfies that the image of $\left\{\zeta \in \mathcal{R}_{C}: \Im(\zeta)=0\right\}$ is again a subset of $\left\{\zeta \in \mathcal{R}_{C}: \Im(\zeta)=0\right\}$, then $f$ admits a real Dulac asymptotic expansion (2.2), and we call $f$ a real Dulac germ or an almost regular germ [I1'91].

The complex Dulac germs appear naturally as corner maps of hyperbolic complex saddles in $\mathbb{C}^{2}$. For example, consider the corner map of a complex saddle

$$
\left\{\begin{array}{l}
z^{\prime}=z+O(2), \\
w^{\prime}=-\alpha_{0} w+O(2),
\end{array}\right.
$$

(where $O(2)$ are complex polynomials in the variables $z$ and $w$ of order at least 2, and $\alpha_{0} \in \mathbb{R}_{>0}$ ), realized between a pair of transversals $\{w=1\} \simeq \widetilde{\mathbb{C}}$ (horizontal) and $\{z=1\} \simeq \widetilde{\mathbb{C}}$ (vertical) with canonical parametrizations as unit disks, or between any analytic reparametrizations of these transversals. By Section 7 in [Lor05] this is a complex Dulac map. Note that the domain of definition of such corner maps is also a standard quadratic domain $\mathcal{R}_{C}$. Indeed, any complex saddle vector field (2.5) is orbitally analytically equivalent to a normal form (see Section $22 \mathrm{C}$ in [IY08]):

$$
\left\{\begin{array}{l}
z^{\prime}=z, \\
w^{\prime}=w\left(-\alpha_{0}+h(z, w)\right),
\end{array}\right.
$$

where $h$ is a complex analytic germ in two variables at $(0,0)$ and $h(z, w)=O(z w)$, as $z, w \rightarrow 0$. Computing a Dulac corner map of a complex saddle (2.6), exactly in the same way as it was done by Ilyashenko for complexified real saddles (see e.g. 
Section 3, Proof of Theorem 7.7 in [Rou98]), we obtain that the domain of definition of the analytic corner map in the logarithmic chart has exponential growth:

$$
\mathcal{E}_{C, M}:=\left\{\zeta \in \mathbb{C}:|\Im(\zeta)| \leq C \mathrm{e}^{M \Re(\zeta)}\right\}, C, M>0,
$$

and it contains standard quadratic domains.

Note that the saddle corner maps defined by foliation (2.6) are not univalued, and that the procedure described in the proof of Theorem 7.7 in [Rou98] provides just one possible determination. Indeed, the time being now a complex variable, one moves along the foliation described by $(2.6)$ from the point $(z, 1)$ on the horizontal transversal to the point $(1, D(z))$ on the vertical transversal in complex time $-\log z=-\log |z|-\mathrm{i} \theta+2 k \pi \mathrm{i}, k \in \mathbb{Z}$. Depending on which determination of the logarithm one chooses (adding $2 k \pi \mathrm{i}, k \in \mathbb{Z}$, to the complex time), one obtains a different determination of the Dulac map $D(z)$. This corresponds to moving from the point $z$ along different paths of the complex foliation, that, when projected to the horizontal or the vertical separatrix, may include circling around the axes or not (resulting in composing $D$ with holonomies of the vertical and horizontal axes, which are non-ramified germs). For more details, see [Lor05]. However, no matter which determination we choose, $D(z)$ is a complex Dulac germ defined on a standard quadratic domain.

In [Il'84] Il'yashenko proves the following quasianalyticity property of Dulac germs: if the Dulac expansion of a real Dulac germ on a standard quadratic domain is just the identity, then the germ itself is equal to the identity. The same property, based on Phragmen-Lindelöf's maximum principle, can be proven similarly for complex Dulac germs, independently of the property of invariance of $\mathbb{R}_{\geq 0}$ which is not necessarily satisfied for complex Dulac germs.

2.2. The main result: Theorem B. Consider an analytic germ at $+\infty$ in the $\zeta$-chart $\mathbb{C}$. We say that $f$ is:

(1) parabolic if $f(\zeta)=\zeta+o(1)$ as $|\zeta| \rightarrow+\infty$, and if $f^{\circ q} \neq$ id for all $q \in \mathbb{N}_{\geq 1}$,

(2) hyperbolic if $f(\zeta)=\zeta+\beta+o(1)$, as $|\zeta| \rightarrow+\infty$, for some $\beta \in \mathbb{C}^{+}$(we can always suppose that this is the case up to replacing $f$ by $f^{-1}$ ).

For all our results, every statement on germs of maps means, as usual, the similar statement for some representative of these germs. Our version of Koenigs' linearization in the setting of hyperbolic complex Dulac germs is the following:

Theorem B (Linearization of hyperbolic complex Dulac germs). Let $f(\zeta)=\zeta+$ $\beta+o(1), \beta \in \mathbb{C}^{+}$, be a hyperbolic complex Dulac germ on a standard quadratic domain $\mathcal{R}_{C}$. Then there exists a unique parabolic germ $\varphi$ satisfying

$$
\varphi \circ f=\varphi+\beta,
$$

on $f$-invariant germs of $\mathcal{R}_{C}$. Moreover, $\varphi$ is a complex parabolic Dulac germ (possibly on a smaller standard quadratic subdomain $\mathcal{R}_{C^{\prime}} \subset \mathcal{R}_{C}$ ). Furthermore, if $f$ is a real Dulac germ, then $\varphi$ is also a real Dulac germ.

Notice that the linearization equation (2.7), written in the $\zeta$-chart, is an Abeltype equation which says that $\varphi$ conjugates $f$ to the translation by $\beta$. Written in the $z$-chart, it would become a Schröder-type equation

$$
\widetilde{\varphi} \circ \widetilde{f}=\lambda \widetilde{\varphi},
$$

where $\widetilde{\varphi}(z):=\exp (-\varphi(-\log z))$ and $\lambda=\exp (-\beta) \in \widetilde{\mathbb{C}}$. 
The proof of Theorem B is in Section 4. In the statement of Theorem B, the linearization of a Dulac germ is done on an invariant germ of a standard quadratic domain. The proof would also go through on every other invariant domain (e.g. constructed from various admissible domains from Subsection 3.1). Quadratic domains are chosen only for convenience, since they are the standard domains for Dulac germs, introduced in [I''84]. In the logarithmic chart, such domains are biholomorphic to $\mathbb{C}^{+}$, and hence the quasianalyticity property holds [Rou98, Il'84].

In Section 3, we prove a version (Theorem A in Subsection 3.3) of this linearization result for less restrictive analytic maps defined on invariant spiraling neighborhoods of the origin of $\widetilde{\mathbb{C}}$ (constructed from their various admissible domains), whose asymptotic behavior is bounded by a particular logarithmic term. This statement, proven via the study of the convergence of the Koenigs sequences, is a generalization to the complex setting of the original Koenigs result and of the theorem of Dewsnap and Fisher in [DF03].

The refinement about the Dulac nature of the linearizing coordinate in the case of (complex) Dulac maps is proven in Section 4. It is based on our results on linearization of (formal) hyperbolic transseries in [PRRS21] and on the resolution of a particular homological equation.

Remark 2.4. Note that the Dulac nature of the linearization $\varphi$ in Theorem B is important: the quasianalyticity result of Ilyashenko [Il'84] for real Dulac germs on standard quadratic domains (that is easily adaptable to complex Dulac germs) implies that the formal linearization $\widehat{\varphi}$ of the real Dulac expansion $\widehat{f}$ of a hyperbolic real Dulac germ $f$ obtained in [PRRS21] (and repeated here in Lemma 4.2) uniquely determines the (unique) analytic linearization $\varphi$ of $f$ on a standard quadratic domain. Therefore it is sufficient to work only with formal series.

\section{Analytic linearization}

The purpose of this section is to show a general complex extension of the results of [DF03]: if a holomorphic map, defined on an appropriate invariant domain of the Riemann surface of the logarithm, has an asymptotic behavior similar to the one described in [DF03], then it can be linearized by a parabolic change of coordinates obtained as the uniform limit of its Koenigs sequence in the logarithmic chart. In particular, we prove in Example (3) below that the standard quadratic domains defined in Section 2 (on which the Dulac maps are usually considered) are particular types of such admissible (invariant) domains for the complex germs mentioned above. The general linearization result of this section will be used in the proof of our main result (Theorem B, Section 4), which asserts the linearizability of hyperbolic Dulac maps by parabolic Dulac changes of coordinates on standard quadratic domains.

In Subsection 3.1, we give the definition of an admissible domain. These provide the main ingredient for producing invariant domains for hyperbolic complex holomorphic germs on $\widetilde{\mathbb{C}}$ with asymptotic behavior of type (3.9), as stated in Proposition 3.4.

In Subsection 3.2, we give several examples of such domains. We show that standard quadratic domains and similar types of domains (bounded by curves of 
any growth $x^{r}, r>0$, at infinity in the logarithmic chart) are invariant for all hyperbolic germs of type (3.9), in particular, for complex hyperbolic Dulac germs. Finally, in Subsection 3.3 we state and prove our linearization result on invariant domains for hyperbolic maps with complex multipliers and an asymptotic behavior as in [DF03] (Theorem A). The computations of this subsection are to some extent motivated by a normalization method for parabolic analytic germs in $(\mathbb{C}, 0)$ described in [Lor98], and by Koenigs' linearization theorem for hyperbolic analytic germs in $(\mathbb{C}, 0)$ described for example in [CG93].

In this section, all the subdomains of the Riemann surface of the logarithm and all the maps defined on these domains are described in the logarithmic chart $\zeta=-\log z$.

3.1. Admissible domains. Recall that $\mathbb{C}^{+}:=\{\zeta \in \mathbb{C}: \Re(\zeta)>0\}$. Let $\beta \in \mathbb{C}^{+}$, $\varepsilon>0$ and $k \in \mathbb{N}$. Let

$$
\begin{aligned}
M_{\varepsilon, k}(x) & :=\frac{1}{x \log x \cdots\left(\log ^{\circ k} x\right)^{1+\varepsilon}} \\
\rho_{\beta, \varepsilon, k}^{ \pm}(x) & :=\Re(\beta) \pm M_{\varepsilon, k}(x), \quad \text { for } x \in\left(\exp ^{\circ k}(0),+\infty\right) .
\end{aligned}
$$

Note that $M_{\varepsilon, k}$ is a positive, strictly decreasing map tending to 0 , as $x \rightarrow+\infty$. Therefore, $\rho_{\beta, \varepsilon, k}^{-}$is a strictly increasing map and $\rho_{\beta, \varepsilon, k}^{+}$is a strictly decreasing map, both tending to $\Re(\beta)$ at infinity. Furthermore, it is known that the series $\sum_{n \in \mathbb{N}} M_{\varepsilon, k}(x+n y)$ converges for every $x, y>0$ (this last fact, which was used in [DF03], will also be used in the proof of Theorem A).

In order to define admissible domains of type $(\beta, \varepsilon, k)$, we first define two functions $h_{l}$ and $h_{u}$, whose graphs bound the domain from "below" and from "above". We distinguish three cases: $\Im(\beta)>0, \Im(\beta)=0$ and $\Im(\beta)<0$ :

(i) Case $\Im(\beta)>0$. Let $t>\exp ^{\circ k}(0)$ such that $\rho_{\beta, \varepsilon, k}^{-}(x)>0$ and $\Im(\beta)-$ $M_{\varepsilon, k}(x)>0, x \in[t,+\infty)$. Let $h_{l}, h_{u}:[t,+\infty) \rightarrow \mathbb{R}$ be any two functions satisfying:

(1) $h_{l}(x)<h_{u}(x), x \in[t,+\infty)$;

(2) $h_{l}$ is a decreasing map on $[t,+\infty)$, or $h_{l}$ is an increasing map with property:

$$
h_{l}\left(x+\rho_{\beta, \varepsilon, k}^{+}(x)\right)-h_{l}(x) \leq \Im(\beta)-M_{\varepsilon, k}(x), x \in[t,+\infty) ;
$$

(3) $h_{u}$ is an increasing map with property:

$$
h_{u}\left(x+\rho_{\beta, \varepsilon, k}^{-}(x)\right)-h_{u}(x) \geq \Im(\beta)+M_{\varepsilon, k}(x), x \in[t,+\infty) .
$$

(ii) Case $\Im(\beta)=0$. Let $t>\exp ^{\circ k}(0)$ such that $\rho_{\beta, \varepsilon, k}^{-}(x)>0, x \in[t,+\infty)$. Let $h_{l}, h_{u}:[t,+\infty) \rightarrow \mathbb{R}$ be any two functions satisfying:

(1) $h_{l}(x)<h_{u}(x), x \in[t,+\infty)$;

(2) $h_{l}$ is a decreasing map with property:

$$
h_{l}\left(x+\rho_{\beta, \varepsilon, k}^{-}(x)\right)-h_{l}(x) \leq-M_{\varepsilon, k}(x), x \in[t,+\infty) ;
$$

(3) $h_{u}$ is an increasing map with property:

$$
h_{u}\left(x+\rho_{\beta, \varepsilon, k}^{-}(x)\right)-h_{u}(x) \geq M_{\varepsilon, k}(x), x \in[t,+\infty) .
$$


(iii) Case $\Im(\beta)<0$. Let $t>\exp ^{\circ k}(0)$ such that $\rho_{\beta, \varepsilon, k}^{-}(x)>0$ and $-\Im(\beta)-$ $M_{\varepsilon, k}(x)>0, x \in[t,+\infty)$. Let $h_{l}, h_{u}:[t,+\infty) \rightarrow \mathbb{R}$ be any two functions satisfying:

(1) $h_{l}(x)<h_{u}(x), x \in[t,+\infty)$;

(2) $h_{l}$ is a decreasing map on $[t,+\infty)$ :

$$
h_{l}\left(x+\rho_{\beta, \varepsilon, k}^{-}(x)\right)-h_{l}(x) \leq \Im(\beta)-M_{\varepsilon, k}(x), x \in[t,+\infty) ;
$$

(3) $h_{u}$ is an increasing map, or a decreasing map with property:

$$
h_{u}\left(x+\rho_{\beta, \varepsilon, k}^{+}(x)\right)-h_{u}(x) \geq \Im(\beta)+M_{\varepsilon, k}(x), x \in[t,+\infty) .
$$

A map $h_{l}:[t,+\infty) \rightarrow \mathbb{R}$ with property (2) is called a lower map of type $(\beta, \varepsilon, k)$. A map $h_{u}:[t,+\infty) \rightarrow \mathbb{R}$ with property (3) is called an upper map of type $(\beta, \varepsilon, k)$. A pair $\left(h_{l}, h_{u}\right)$ of maps $h_{l}, h_{u}:[t,+\infty) \rightarrow \mathbb{R}$, satisfying conditions (1) - (3) above, is called a lower-upper pair of type $(\beta, \varepsilon, k)$. Notice that the opposite of an upper map of type $(\beta, \varepsilon, k)$ is a lower map of type $(\beta, \varepsilon, k)$.

Finally, let

$$
D_{h_{l}, h_{u}}:=\left\{\zeta \in \mathbb{C}^{+}: \operatorname{Re} \zeta \geq t, h_{l}(\operatorname{Re} \zeta)<\operatorname{Im} \zeta<h_{u}(\operatorname{Re} \zeta)\right\} .
$$

Definition 3.1 (Admissible domain). Let $\beta \in \mathbb{C}^{+}, \varepsilon>0$ and $k \in \mathbb{N}$. A domain of type $(\beta, \varepsilon, k)$ (or $(\beta, \varepsilon, k)$-domain) is defined as a union of an arbitrary nonempty collection of subsets of the form $D_{h_{l}, h_{u}} \subseteq \mathbb{C}$ defined above. Similarly, a subset $D \subseteq \mathbb{C}$ which contains a $(\beta, \varepsilon, k)$-domain is called an admissible domain of type $(\beta, \varepsilon, k)$ (or $(\beta, \varepsilon, k)$-admissible domain).

Remark 3.2. It follows from Definition 3.1 that an arbitrary union of domains of type $(\beta, \varepsilon, k)$ is again a domain of type $(\beta, \varepsilon, k)$.

\subsection{Examples.}

In this subsection, we fix $\beta \in \mathbb{C}^{+}, \varepsilon>0$ and $k \in \mathbb{N}$. We give here several examples of upper (lower) maps of type $(\beta, \varepsilon, k)$ and of $(\beta, \varepsilon, k)$-admissible domains.

In particular, the fact that a standard quadratic domain as in $(2.1)$ is $(\beta, \varepsilon, k)$ admissible is proven in Example (3).

We first provide a general technical sufficient condition under which a map $h$ is an upper or a lower map. This condition is then used in the following Examples (2) and (4).

Upper map condition in case $\Im(\beta) \geq 0$. Let $t>\exp ^{\circ k}(0)$ such that $\rho_{\beta, \varepsilon, k}^{-}(t)>0$. Note that $\rho_{\beta, \varepsilon, k}^{-}(t) \leq \rho_{\beta, \varepsilon, k}^{-}(x)$, for $x \in[t,+\infty)$. Let $h \in \mathcal{C}^{n}([t,+\infty))$, for some $n \in \mathbb{N}_{\geq 1}$, be an increasing map. Suppose that there exists a positive number $0<\rho<\rho_{\beta, \varepsilon, k}^{-}(t)$ such that

$$
\sum_{i=1}^{n} \frac{h^{(i)}(x)}{i !} \rho^{i} \geq \Im(\beta)+M_{\varepsilon, k}(x), \text { for all } x \geq t,
$$

and that $h^{(n)}:[t,+\infty) \rightarrow \mathbb{R}$ is increasing. Then $h$ is an upper map of type $(\beta, \varepsilon, k)$.

This can be seen as follows. Since $h$ and $h^{(n)}$ are increasing, it follows from Taylor's theorem and then (3.3) that

$$
\begin{aligned}
h\left(x+\rho_{\beta, \varepsilon, k}^{-}(x)\right)-h(x) & \geq h(x+\rho)-h(x) \geq \sum_{i=1}^{n} \frac{h^{(i)}(x)}{i !} \rho^{i} \\
& \geq \Im(\beta)+M_{\varepsilon, k}(x), x \in[t,+\infty) .
\end{aligned}
$$


Upper map condition in case $\Im(\beta)<0$. Let $t>\exp ^{\circ k}(0)$. Suppose that $h:[t,+\infty) \rightarrow \mathbb{R}$ is either an increasing map, or a decreasing map belonging to $\mathcal{C}^{n}([t,+\infty))$ for some $n \in \mathbb{N}_{\geq 1}$, which satisfies, for some positive number $\rho>$ $\rho_{\beta, \varepsilon, k}^{+}(t)$ :

$$
\sum_{i=1}^{n} \frac{h^{(i)}(x)}{i !} \rho^{i} \geq \Im(\beta)+M_{\varepsilon, k}(x), \text { for all } x \geq t,
$$

and that $h^{(n)}:[t,+\infty) \rightarrow \mathbb{R}$ is increasing. Then $h$ is an upper map of type $(\beta, \varepsilon, k)$.

Lower map condition in case $\Im(\beta)>0$. Let $t>\exp ^{\circ k}(0)$ such that $\Im(\beta)-$ $M_{\varepsilon, k}(t)>0$. Then, $\Im(\beta)-M_{\varepsilon, k}(x)>0, x \in[t,+\infty)$. Similarly as above, if $h$ is either decreasing on $[t,+\infty)$, or increasing belonging to $\mathcal{C}^{n}([t,+\infty))$ for some $n \in \mathbb{N}_{\geq 1}$, and satisfying the property

$$
\sum_{i=1}^{n} \frac{h^{(i)}(x)}{i !} \rho^{i} \leq \Im(\beta)-M_{\varepsilon, k}(x), \text { for all } x \geq t,
$$

for some $\rho>\rho_{\beta, \varepsilon, k}^{+}(t)$, and with $h^{(n)}$ decreasing on $[t,+\infty)$, it follows that $h$ is a lower map of type $(\beta, \varepsilon, k)$.

Lower map condition in case $\Im(\beta) \leq 0$. Let $t>\exp ^{\circ k}(0)$ such that $\rho_{\beta, \varepsilon, k}^{-}(t)>$ 0 . If $h$ is a decreasing map belonging to $\mathcal{C}^{n}([t,+\infty))$ for some $n \in \mathbb{N}_{\geq 1}$, and satisfying the property

$$
\sum_{i=1}^{n} \frac{h^{(i)}(x)}{i !} \rho^{i} \leq \Im(\beta)-M_{\varepsilon, k}(x), \text { for all } x \geq t,
$$

for some $0<\rho<\rho_{\beta, \varepsilon, k}^{-}(t)$, and if $h^{(n)}$ is decreasing, it follows that $h$ is a lower map of type $(\beta, \varepsilon, k)$.

Example (1). (Sufficient condition for upper/lower maps)

Here, $\Im(\beta) \geq 0$. Let $t>\exp ^{\circ k}(0)$ be such that $\rho_{\beta, \varepsilon, k}^{-}(t)>0$. Let $h:[t,+\infty) \rightarrow \mathbb{R}$ be an increasing map of class $\mathcal{C}^{1}$, such that:

1. $h^{\prime}:[t,+\infty) \rightarrow \mathbb{R}$ tends to $\lambda^{\prime}>\frac{\Im(\beta)}{\Re(\beta)}$, as $x \rightarrow+\infty$. Then, since $M_{\varepsilon, k}(x) \rightarrow 0$ and $\rho_{\beta, \varepsilon, k}^{-} \rightarrow \Re(\beta)$ as $x \rightarrow+\infty$, there exists $t^{\prime} \geq t$ sufficiently large such that

$$
h^{\prime}(x) \geq \frac{\Im(\beta)+M_{\varepsilon, k}\left(t^{\prime}\right)}{\rho_{\beta, \varepsilon, k}^{-}\left(t^{\prime}\right)},
$$

for every $x \geq t^{\prime}$. Since $M_{\varepsilon, k}$ is decreasing and $\rho_{\beta, \varepsilon, k}^{-}$is increasing, for every $v \in(0,1)$ and $x>t^{\prime}$,

$$
h^{\prime}\left(x+v \rho_{\beta, \varepsilon, k}^{-}(x)\right) \cdot \rho_{\beta, \varepsilon, k}^{-}(x) \geq \Im(\beta)+M_{\varepsilon, k}(x) .
$$

Hence, by the Mean Value Theorem, the restriction $\left.h\right|_{\left[t^{\prime},+\infty\right\rangle}$ is an upper map of type $(\beta, \varepsilon, k)$.

2. $h^{\prime}:[t,+\infty) \rightarrow \mathbb{R}$ tends to $+\infty$, as $x \rightarrow+\infty$. Then we choose $t^{\prime} \geq t$ sufficiently large such that (3.6) holds. The restriction $\left.h\right|_{\left[t^{\prime},+\infty\right)}$ is therefore an upper map of type $(\beta, \varepsilon, k)$.

In the case $\Im(\beta)<0$, any increasing $h$ on $[t,+\infty)$ is an upper map of type $(\beta, \varepsilon, k)$.

Analogously, a similar sufficient condition can be deduced for lower maps of type $(\beta, \varepsilon, k)$. 
Example (2). (Maps of type $h(x) \sim x^{r}, r>0$ )

1. Case $r>1$. Let $h:[t,+\infty) \rightarrow \mathbb{R}, t>0$, be an increasing map of class $\mathcal{C}^{1}$ such that ${ }^{1}$

$$
h(x) \sim a x^{r}, h^{\prime}(x) \sim a r x^{r-1}, a>0, r>1, x \rightarrow+\infty .
$$

By Example (1.2), there exists $t>0$ big enough such that $h$ is an upper map of type $(\beta, \varepsilon, k)$.

Let $h:[t,+\infty) \rightarrow \mathbb{R}, t>0$, be a decreasing $\mathcal{C}^{1}$ map , such that

$$
h(x) \sim-a x^{r}, h^{\prime}(x) \sim-a r x^{r-1}, a>0, r>1, x \rightarrow+\infty .
$$

By a similar argument, there exists $t>0$ big enough such that $h$ is a lower map of type $(\beta, \varepsilon, k)$.

2. Case $r=1$. Let $h:[t,+\infty) \rightarrow \mathbb{R}, t>0$, be an increasing $\mathcal{C}^{1}$ map such that

$$
h(x) \sim a x, h^{\prime}(x) \sim a, a>0, x \rightarrow+\infty .
$$

Then, by Example (1.1), if $\Im(\beta)<0$, or if $\Im(\beta) \geq 0$ and $\frac{\Im(\beta)}{\Re(\beta)}<a$, there exists $t>0$ big enough such that $h$ is an upper map of type $(\beta, \varepsilon, k)$. Let $h:[t,+\infty) \rightarrow \mathbb{R}$, $t>0$, be a decreasing $\mathcal{C}^{1}$ map satisfying $h(x) \sim-a x, h^{\prime}(x) \sim-a, a>0, x \rightarrow+\infty$. Similarly, if $\Im(\beta)>0$, or if $\Im(\beta) \leq 0$ and $\frac{\Im(\beta)}{\Re(\beta)}>-a$, there exists big enough $t>0$ such that $h$ is a lower map of type $(\beta, \varepsilon, k)$.

3. Case $0<r<1$. The sufficient condition of Example (1) is not satisfied for $0<r<1$. However, suppose that $h$ is increasing (resp. decreasing) of class $\mathcal{C}^{2}$, satisfying (3.7) (resp. (3.8)) with $r<1$, with the additional property that $h^{\prime \prime}(x) \sim \operatorname{ar}(r-1) x^{r-2}\left(\right.$ resp. $\left.h^{\prime \prime}(x) \sim-\operatorname{ar}(r-1) x^{r-2}\right)$ and $h^{\prime \prime}$ is increasing (resp. decreasing). It follows from conditions (3.3) and (3.5), in a similar way as in Example (4) below, that if $\Im(\beta)=0$, then $h$ is an upper (resp. lower) map of type $(\beta, \varepsilon, k), \varepsilon>0, k \in \mathbb{N}$.

\section{Example (3). (Standard quadratic domains)}

For $C>0$, let $\mathcal{R}_{C} \subseteq \mathbb{C}$ be the standard quadratic domain defined in (2.1). The upper half of the boundary of $\mathcal{R}_{C}$ is described by a smooth function which satisfies sufficient conditions of Example (1.2) (and the lower half satisfies the symmetric statement), hence such a domain is admissible.

Indeed, a direct computation shows that the boundary

$$
\partial\left(\mathcal{R}_{C} \cap\{\zeta \in \mathbb{C}: \operatorname{Im} \zeta \geq 0\}\right)
$$

can be parameterized by:

$$
\begin{aligned}
& r \rightarrow x(r)+\mathrm{i} \cdot y(r)=C \sqrt[4]{r^{2}+1} \cos \left(\frac{1}{2} \operatorname{arctg} r\right)+ \\
&+\mathrm{i} \cdot\left(r+C \sqrt[4]{r^{2}+1} \sin \left(\frac{1}{2} \operatorname{arctg} r\right)\right), r \in[0,+\infty) .
\end{aligned}
$$

Note that $y:[0,+\infty) \rightarrow \mathbb{R}$ is strictly increasing. Let $t>0$ be such that $x(t)>$ $\exp ^{\circ k}(0)$ and $x$ is strictly increasing on $[t,+\infty)$. Therefore, $h_{u}:=y \circ x^{-1}$ is strictly increasing on $[x(t),+\infty)$. By direct computation, if can be shown that the derivative of $h_{u}$ on $[x(t),+\infty)$ tends to $+\infty$, as $x \rightarrow+\infty$. Therefore, by Example (1.2), there exists $x^{\prime}>0$ such that the restriction $\left.h_{u}\right|_{\left[x^{\prime},+\infty\right)}$ is an upper map of type $(\beta, \varepsilon, k)$.

\footnotetext{
${ }^{1}$ We write $f \sim g, x \rightarrow \infty$, if $\lim _{x \rightarrow \infty} \frac{f(x)}{g(x)}=1$.
} 
A similar argument can be repeated to show that an appropriate restriction of the lower boundary of $\mathcal{R}_{C}$ is the graph of a lower map of type $(\beta, \varepsilon, k)$.

Example (4). (Logarithmic upper/lower maps)

Logarithmic upper maps. Let $\beta \in \mathbb{C}^{+}$such that $\Im(\beta)=0$. Let $h:[t,+\infty) \rightarrow \mathbb{R}$, $h(x):=(\log x)^{\delta}, \delta \in \mathbb{R}_{>0}, t \in \mathbb{R}_{>1}$. Note that:

$$
\begin{aligned}
h^{\prime}(x) & =\frac{\delta(\log x)^{\delta}}{x \log x}, \\
h^{\prime \prime}(x) & =\frac{\delta(\log x)^{\delta}}{x \log x} \cdot\left(\frac{\delta-1}{x \log x}-\frac{1}{x}\right), \quad x \geq t .
\end{aligned}
$$

For every $0<\rho<\rho_{\beta, \varepsilon, k}^{-}$, there exists $t>\exp ^{\circ k}(0)$ big enough such that

$$
\begin{aligned}
& h^{\prime}(x) \rho+\frac{1}{2} h^{\prime \prime}(x) \rho^{2}=\frac{1}{x \log x} \cdot \rho \delta(\log x)^{\delta} \cdot\left(1+\frac{\rho(\delta-1)}{2 x \log x}-\frac{\rho}{2 x}\right) \\
& \geq \frac{1}{x \log x} \cdot \frac{1}{\log ^{\circ 2} x \cdots\left(\log ^{\circ k} x\right)^{1+\varepsilon}}=M_{\varepsilon, k}(x),
\end{aligned}
$$

for $x \geq t$. It can be proven that we can take $t>\exp ^{\circ k}(0)$ big enough such that $h^{\prime \prime \prime}(x)>0$, for each $x \geq t$. This implies that restriction of $h^{\prime \prime}$ on $[t,+\infty)$ is an increasing map. Therefore, since $h$ is increasing, it follows from sufficient upper map condition (3.3) that there exists $t>\exp ^{\circ k}(0)$ such that the restriction $\left.\left.h\right|_{[t,+\infty}\right)$ is an upper map of type $(\beta, \varepsilon, k)$.

Logarithmic lower maps. Let $\beta \in \mathbb{C}^{+}$such that $\Im(\beta)=0$ and let $h$ be as defined above. It follows that $g:[t,+\infty) \rightarrow \mathbb{R}$, defined by $g(x):=-h(x), x \in[t,+\infty)$, is a lower map of type $(\beta, \varepsilon, k)$.

\subsection{Linearization theorem for holomorphic maps on spiraling domains: Theorem A.}

Notation 3.3. For every set $D \subseteq \mathbb{C}$ and $R>0$, let

$$
D_{R}:=D \cap([R,+\infty) \times \mathbb{R}) \subseteq \mathbb{C} .
$$

Note that, if $D \subseteq \mathbb{C}$ is a (admissible) domain of type $(\beta, \varepsilon, k)$, then $D_{R}$ is a (admissible) domain of type $(\beta, \varepsilon, k)$.

For every admissible domain $D$ of type $(\beta, \varepsilon, k)$, denote by $\bar{D}$ its maximal subdomain of type $(\beta, \varepsilon, k)$. That is, by Remark $3.2, \bar{D}$ is defined as the union of all subdomains of type $(\beta, \varepsilon, k)$ of $D$.

Denote by $D^{f}$ the maximal $f$-invariant subdomain of an admissible domain $D \subseteq \mathbb{C}$ (i.e. the union of all $f$-invariant subdomains of $D$ ).

Finally, for an admissible domain $D$, define

$$
D_{R}^{f}:=\left(D^{f}\right)_{R}, \bar{D}_{R}:=(\bar{D})_{R}, R>0 .
$$

We extend the above definition to the case $D=\mathbb{C}^{+}$by stipulating that $\bar{D}_{R}=$ $\left\{\zeta \in \mathbb{C}^{+}: \Re(\zeta)>R\right\}$. For an admissible domain $D \subseteq \mathbb{C}$, the maximal $f$-invariant subdomain $D^{f} \subseteq D$ can be, in general, empty. The following proposition gives sufficient conditions such that $D^{f}$ and $D_{R}^{f}$, for every $R>0$, are non-empty. 
Proposition 3.4. Let $\beta \in \mathbb{C}^{+}, \varepsilon>0$ and $k \in \mathbb{N}$. Let $D \subseteq \mathbb{C}^{+}$be an admissible domain of type $(\beta, \varepsilon, k)$. Let $f: D_{C} \rightarrow \mathbb{C}, C>\exp ^{\circ k}(0)$, be an analytic map, such that

$$
f(\zeta)=\zeta+\beta+o\left(\zeta^{-1} \boldsymbol{L}_{1}^{-1} \cdots \boldsymbol{L}_{k}^{-(1+\varepsilon)}\right), \quad \text { as } \Re(\zeta) \rightarrow+\infty \text { uniformly on } D_{C} .
$$

Here,

$$
\boldsymbol{L}_{1}:=\log (\zeta), \ldots, \boldsymbol{L}_{k}:=\log \left(\boldsymbol{L}_{k-1}\right),
$$

where $\log$ represents the principal branch of the logarithm ${ }^{2}$. Then, for every $R>C$ sufficiently large, the domain $\bar{D}_{R}$ is f-invariant. In particular, $\bar{D}_{R} \subseteq D_{R}^{f}$ and $D_{R}^{f} \neq \emptyset$.

Proof. By asymptotics (3.9),

$$
\lim _{\Re(\zeta) \rightarrow+\infty} \frac{f(\zeta)-(\zeta+\beta)}{\zeta^{-1} \boldsymbol{L}_{1}^{-1} \cdots \boldsymbol{L}_{k}^{-(1+\varepsilon)}}=0,
$$

uniformly on $D$.

Let $\rho_{\beta, \varepsilon, k}^{ \pm}$and $M_{\varepsilon, k}$ be as defined in (3.1). By (3.10), there exists $R>\exp ^{\circ k}(0)$ such that $\rho_{\beta, \varepsilon, k}^{-}(R)>0, \rho_{\beta, \varepsilon, k}^{-}$is increasing on $[R,+\infty)$ and, for all $\zeta \in D_{R}$,

$$
|f(\zeta)-(\zeta+\beta)| \leq \frac{1}{\left|\zeta \boldsymbol{L}_{1} \cdots \boldsymbol{L}_{k}^{1+\varepsilon}\right|} .
$$

Since $R>\exp ^{\circ k}(0)$ and $|\log \zeta| \geq \log |\zeta| \geq \log (|\Re(\zeta)|)=\log (\Re(\zeta))$, we inductively get:

$$
\left|\boldsymbol{L}_{m}\right| \geq \log ^{\circ m}(\Re(\zeta)), \text { for } 1 \leq m \leq k, \zeta \in D_{R} .
$$

Now, by (3.11) and (3.12), we get, for $\zeta \in D_{R}$ :

$$
|f(\zeta)-(\zeta+\beta)| \leq \frac{1}{\Re(\zeta) \cdot \log (\Re(\zeta)) \cdots\left(\log ^{\circ k}(\Re(\zeta))\right)^{1+\varepsilon}} .
$$

Therefore, for $\zeta \in D_{R}$ :

$$
\begin{aligned}
\Re(f(\zeta))-\Re(\zeta) & \geq \Re(\beta)-\frac{1}{\Re(\zeta) \cdot \log (\Re(\zeta)) \cdots\left(\log ^{\circ k}(\Re(\zeta))\right)^{1+\varepsilon}} \\
& =\rho_{\beta, \varepsilon, k}^{-}(\Re(\zeta)), \\
\Re(f(\zeta))-\Re(\zeta) & \leq \Re(\beta)+\frac{1}{\Re(\zeta) \cdot \log (\Re(\zeta)) \cdots\left(\log ^{\circ k}(\Re(\zeta))\right)^{1+\varepsilon}} \\
& =\rho_{\beta, \varepsilon, k}^{+}(\Re(\zeta)),
\end{aligned}
$$

and

$$
\begin{aligned}
\Im(f(\zeta))-\Im(\zeta) & \geq \Im(\beta)-\frac{1}{\Re(\zeta) \cdot \log (\Re(\zeta)) \cdots\left(\log ^{\circ k}(\Re(\zeta))\right)^{1+\varepsilon}} \\
& =\Im(\beta)-M_{\varepsilon, k}(\Re(\zeta)),
\end{aligned}
$$

\footnotetext{
${ }^{2}$ Note here that, for $C>\exp ^{\circ k}(0)$, the iterated logarithms $\boldsymbol{L}_{1}, \ldots, \boldsymbol{L}_{k}$ are well-defined on $D_{C}$ (using only the principal branch of the logarithm), since $\Re(\zeta)>\exp ^{\circ k}(0)$.
} 


$$
\begin{aligned}
\Im(f(\zeta))-\Im(\zeta) & \leq \Im(\beta)+\frac{1}{\Re(\zeta) \cdot \log (\Re(\zeta)) \cdots\left(\log ^{\circ k}(\Re(\zeta))\right)^{1+\varepsilon}} \\
& =\Im(\beta)+M_{\varepsilon, k}(\Re(\zeta)) .
\end{aligned}
$$

Since $\rho_{\beta, \varepsilon, k}^{-}$is an increasing function, it follows that $\rho_{\beta, \varepsilon, k}^{-}(\Re(\zeta)) \geq \rho_{\beta, \varepsilon, k}^{-}(R)>0$, for every $\zeta \in D_{R}$. Let, for $\zeta \in D_{R}$,

$$
\begin{aligned}
\mathcal{S}_{\beta, \varepsilon, k}(\zeta) & :=\left[\Re(\zeta)+\rho_{\beta, \varepsilon, k}^{-}(\Re(\zeta)), \Re(\zeta)+\rho_{\beta, \varepsilon, k}^{+}(\Re(\zeta))\right] \\
& \times\left[\Im(\zeta)+\Im(\beta)-M_{\varepsilon, k}(\Re(\zeta)), \Im(\zeta)+\Im(\beta)+M_{\varepsilon, k}(\Re(\zeta))\right] .
\end{aligned}
$$

By (3.14)-(3.17), we get that, for $\zeta \in D_{R}$,

$$
f(\zeta) \in \mathcal{S}_{\beta, \varepsilon, k}(\zeta)
$$

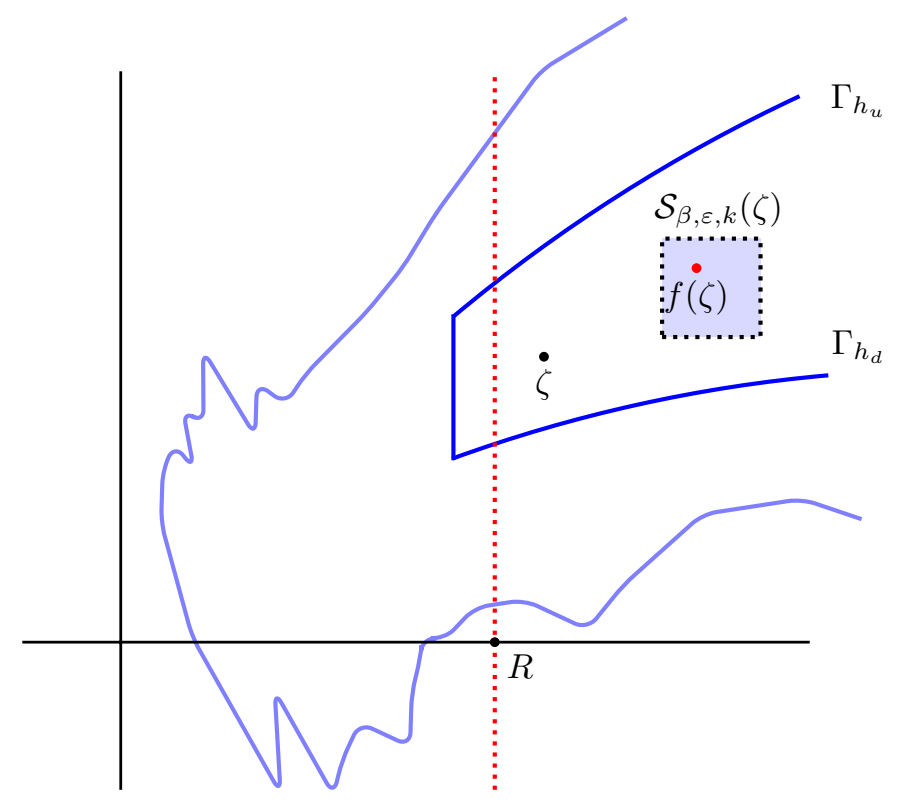

FiguRe 2. For $R$ sufficiently large, $\mathcal{S}_{\beta, \varepsilon, k}(\zeta) \subseteq \bar{D}_{R}, \zeta \in \bar{D}_{R}$.

Now take $\zeta \in \bar{D}_{R}$. It is left to prove that then $f(\zeta) \in \bar{D}_{R}$, that is, that $\bar{D}_{R}$ is $f$ invariant. By definition of $\bar{D}_{R}$, there exists a $(\beta, \varepsilon, k)$-domain $\left(D_{h_{l}, h_{u}}\right)_{R} \subseteq \bar{D}_{R}$, such that $\zeta \in\left(D_{h_{l}, h_{u}}\right)_{R}$. Now, by properties (2) and (3) in the definition of lower-upper pair of type $(\beta, \varepsilon, k)$, it follows that $\mathcal{S}_{\beta, \varepsilon, k}(\zeta) \subseteq D_{h_{l}, h_{u}}$, see Figure 2. Therefore, $f(\zeta) \in D_{h_{l}, h_{u}} \subseteq \bar{D}$. Since $\zeta \in D_{R}$ and $\rho_{\beta, \varepsilon, k}^{-}(\Re(\zeta))>0$ for $\zeta \in D_{R}$, by (3.14) it follows that $\Re(f(\zeta))>\Re(\zeta) \geq R$. Therefore, $f \in \bar{D}_{R}$.

We now state the main result of this section, which establishes the convergence of Koenigs' sequence for a holomorphic map with a logarithmic-type bound on asymptotic behavior on an admissible domain: 
Theorem A. Let $\beta \in \mathbb{C}^{+}, \varepsilon>0, k \in \mathbb{N}$. Let $D \subseteq \mathbb{C}^{+}$be an admissible domain of type $(\beta, \varepsilon, k)$. For $C>\exp ^{\circ k}(0)$, let $f: D_{C} \rightarrow \mathbb{C}$ be an analytic map such that

$$
f(\zeta)=\zeta+\beta+o\left(\zeta^{-1} \boldsymbol{L}_{1}^{-1} \cdots \boldsymbol{L}_{k}^{-(1+\varepsilon)}\right), \quad \text { as } \Re(\zeta) \rightarrow+\infty \text { uniformly on } D_{C} \text {. }
$$

Here, the iterated logarithms $\boldsymbol{L}_{1}, \ldots \boldsymbol{L}_{k}$ are defined as in Proposition 3.4. Then:

(i) (Existence) For a sufficiently large $R>\exp ^{\circ k}(0)$ there exists an analytic linearizing map $\varphi$ on the $f$-invariant subdomain $D_{R}^{f} \subseteq D$. That is, $\varphi$ satisfies

$$
(\varphi \circ f)(\zeta)=\varphi(\zeta)+\beta, \text { for all } \zeta \in D_{R}^{f}
$$

Moreover, $\varphi$ is the uniform limit on $D_{R}^{f}$ of the Koenigs sequence

$$
\left(f^{\circ n}-n \beta\right)_{n} \text {. }
$$

(ii) If $D_{R}^{f} \cap\left\{\zeta \in \mathbb{C}^{+}: \Im(\zeta)=0\right\}$ is $f$-invariant, so is $\varphi$-invariant.

(iii) (Asymptotics) The linearization $\varphi$ is tangent to identity, i.e. $\varphi(\zeta)=\zeta+o(1)$, uniformly on $D_{R}^{f} \subseteq \mathbb{C}^{+}$, as $\Re(\zeta) \rightarrow+\infty$.

In particular, $\varphi(\zeta)=\zeta+o\left(\boldsymbol{L}_{k}^{-\nu}\right)$, for every $\nu \in(0, \varepsilon)$, uniformly as $\Re(\zeta) \rightarrow$ $+\infty$, on every subdomain $D_{h_{l}, h_{u}} \subseteq D_{R}^{f}$ such that $h_{l}(x)=O(x)$ and $h_{u}(x)=$ $O(x), x \rightarrow+\infty$.

(iv) (Uniqueness) Let $\psi: D_{1} \rightarrow \mathbb{C}$, be a linearization of $f$ on an $f$-invariant subset $D_{1} \subseteq D$, such that $\psi(\zeta)=\zeta+o(1)$ uniformly on $D_{1}$, as $\Re(\zeta) \rightarrow+\infty$. Then $\psi \equiv \varphi$ on $\left(D_{1}\right)_{R}$.

Remark 3.5.

(1) Theorem A can be seen as a generalization to complex domains, expressed in the logarithmic chart $\zeta=-\log (z)$, of the results proved in Dewsnap-Fisher [DF03] for real maps.

(2) Note that the condition (3.18) for linearizability is natural. It was indeed proven in [PRRS21] that a hyperbolic logarithmic formal transseries $\widehat{f} \in \mathcal{L}_{k}$ (i.e. with monomials in variables $z, \boldsymbol{\ell}_{1}, \ldots, \boldsymbol{\ell}_{k}$, where $\boldsymbol{\ell}_{1}:=-\frac{1}{\log z}$, and inductively $\boldsymbol{\ell}_{i}:=$ $\boldsymbol{\ell}_{1} \circ \boldsymbol{\ell}_{i-1}$, for $\left.2 \leq i \leq k\right), k \in \mathbb{N}_{\geq 1}$, is formally linearizable if and only if the leading monomial of $\widehat{\widehat{f}}-\lambda z$ is a logarithmic monomial of type $o\left(z \boldsymbol{\ell}_{1} \cdots \boldsymbol{\ell}_{m}^{1+\varepsilon}\right)$ for some $m \in \mathbb{N}_{\geq 1}, 1 \leq m \leq k$, and $\varepsilon>0$. On the other hand, the monomials up to $z \ell_{1} \cdots \ell_{k}$ (included) cannot be eliminated from the normal form of $\widehat{f}$.

Example (Examples for Theorem A). Although Theorem A may be applied to any function satisfying the condition (3.18), we illustrate how this theorem works on some examples of functions that have an expansion in the logarithmic scale.

(1) Let

$$
f(\zeta)=\zeta+2+3 \pi \mathrm{i}+\zeta^{-1} \boldsymbol{L}_{1}^{-2}+\zeta^{-2} \boldsymbol{L}_{2}^{2}+\frac{\mathrm{e}^{-\zeta}}{1-\mathrm{e}^{-\zeta} \boldsymbol{L}_{1}} .
$$

By Proposition 3.4, $f$ is well-defined on a domain $D_{R} \subseteq \mathbb{C}^{+}$where $D$ is an $(\beta, \varepsilon, 2)$ admissible domain, with $\beta=2+3 \pi \mathrm{i}$ and $\varepsilon>0$, and $R>\exp (\exp (0))=\mathrm{e}$. Consider for example any standard quadratic domain $D$ as in Example (3), intersected with the right half plane $\left\{\zeta \in \mathbb{C}^{+}: \Re(\zeta)>R\right\}$. By Theorem A, for sufficiently large $R>\mathrm{e}, D_{R}$ is $f$-invariant and $f$ is linearizable by a tangent to the identity change $\varphi(\zeta)=\zeta+o(1)$, analytic on $D_{R}$ :

$$
\varphi \circ f=\varphi+2+3 \pi \mathrm{i} \text { on } D_{R} .
$$


(2) Let

$$
\begin{aligned}
f(\zeta)=\zeta+2+3 \pi \mathrm{i}+\boldsymbol{L}_{1}^{-1}+\zeta^{-1} \boldsymbol{L}_{2}^{-1} \boldsymbol{L}_{3}+ & 4 \zeta^{-1} \boldsymbol{L}_{1}^{-1} \boldsymbol{L}_{2}^{-1} \boldsymbol{L}_{3}+ \\
& +\zeta^{-2}+\mathrm{e}^{-\zeta} \boldsymbol{L}_{1}^{2}+\mathrm{e}^{-2 \zeta}
\end{aligned}
$$

on some $D_{R}$, where $D$ is a $(\beta, \varepsilon, 3)$-admissible domain with $\beta=2+3 \pi \mathrm{i}, \varepsilon>0$, and $R>\exp ^{\circ 3}(0)$ sufficiently big so that $\bar{D}_{R}$ is invariant for $f$.

By [PRRS21] $]^{3}$, if we consider $f$ as a formal series in variables $\zeta^{-1}, \boldsymbol{L}_{1}, \boldsymbol{L}_{2}, \boldsymbol{L}_{3}$ and $\mathrm{e}^{-\zeta}, f$ is not formally linearizable by any logarithmic transseries. Nevertheless, it can be formally reduced to a normal form

$$
f_{0}(\zeta)=\zeta+2+3 \pi \mathrm{i}+\boldsymbol{L}_{1}^{-1}+\zeta^{-1} \boldsymbol{L}_{2}^{-1} \boldsymbol{L}_{3}+4 \zeta^{-1} \boldsymbol{L}_{1}^{-1} \boldsymbol{L}_{2}^{-1} \boldsymbol{L}_{3}
$$

where the terms strictly before the term $\zeta^{-1} \boldsymbol{L}_{1}^{-1} \boldsymbol{L}_{2}^{-1} \boldsymbol{L}_{3}^{-1-\varepsilon}$, for any $\varepsilon>0$, cannot be eliminated from the normal form by formal changes of variables.

Note that Theorem A does not claim the analytic linearization of $f$ on $\bar{D}_{R}$, since $f$ does not satisfy (3.18). Although Theorem A is not an "if and only if" statement, formal non-linearizability is a good indication that analytic linearization of $f$ on $\bar{D}_{R}$ may not be possible. We conjecture instead that the proof of Theorem A can be adapted to the analytic normalization of $f$ to $f_{0}$ on $\bar{D}_{R}$ in this case. We omit it here, since the use of techniques similar to the proof of Theorem A is to be expected, but with more technical complications.

\section{Proof of Theorem A.}

(i) By Proposition 3.4, there exists $R>\exp ^{\circ k}(0)$ sufficiently large, such that $\bar{D}_{R} \subseteq D_{R}^{f}$, where $D^{f}$ is the maximal $f$-invariant subdomain of $D \subseteq \mathbb{C}^{+}$. Therefore, $D_{R^{\prime}}^{f} \neq \emptyset$, for all $R^{\prime} \geq R$. Let $\zeta \in D_{R}^{f}$ and let $\rho_{\beta, \varepsilon, k}^{ \pm}$be the increasing functions defined in (3.1). From (3.14), for $\zeta \in D_{R}^{f}$ with $R$ sufficiently large, since $\rho_{\beta, \varepsilon, k}^{-}$is increasing on $[R,+\infty)$, it follows that:

$$
\Re\left(f^{\circ n}(\zeta)\right) \geq \Re(\zeta)+n \rho_{\beta, \varepsilon, k}^{-}(\Re(\zeta)) \geq R+n \rho_{\beta, \varepsilon, k}^{-}(R), n \in \mathbb{N}_{\geq 1} .
$$

By (3.13), for $\zeta \in D_{R}^{f}$ it holds that:

$$
|f(\zeta)-(\zeta+\beta)| \leq \frac{1}{\Re(\zeta) \cdot \log (\Re(\zeta)) \cdots\left(\log ^{\circ k}(\Re(\zeta))\right)^{1+\varepsilon}}=M_{\varepsilon, k}(\Re(\zeta)) .
$$

\footnotetext{
${ }^{3}$ Written in the $z$-chart, $z=\mathrm{e}^{-\zeta}, f$ given in (3.20) is a series of monomials in $z, \ell_{1}, \ell_{2}, \ell_{3}$ of the form $\tilde{f}(z):=\mathrm{e}^{-f(-\log z)}=\mathrm{e}^{-2-3 \pi \mathrm{i}} z\left(1+\left(\mathrm{e}^{-\ell_{2}}-1\right)-\boldsymbol{\ell}_{1} \boldsymbol{\ell}_{3} \boldsymbol{\ell}_{4}^{-1}-3 \boldsymbol{\ell}_{1} \boldsymbol{\ell}_{2} \boldsymbol{\ell}_{3} \boldsymbol{\ell}_{4}^{-1}\right)+o\left(z \boldsymbol{\ell}_{1} \boldsymbol{\ell}_{2} \boldsymbol{\ell}_{3} \boldsymbol{\ell}_{4}^{1+\varepsilon}\right)$, $\varepsilon>0$. Also, in the $z$-chart, $f_{0}$ from $(3.21)$ is of the form $\tilde{f}_{0}(z):=\mathrm{e}^{-f_{0}(-\log z)}=\mathrm{e}^{-2-3 \pi \mathrm{i}} z(1+$ $\left.\left(\mathrm{e}^{-\ell_{2}}-1\right)-\boldsymbol{\ell}_{1} \ell_{3} \ell_{4}^{-1}-3 \boldsymbol{\ell}_{1} \ell_{2} \ell_{3} \ell_{4}^{-1}\right)+o\left(z \boldsymbol{\ell}_{1} \ell_{2} \ell_{3} \ell_{4}^{1+\varepsilon}\right)$. By [PRRS21], $\tilde{f}$ can be formally reduced to $\tilde{f}_{0}$; therefore, $f$ can be formally reduced to $f_{0}$ (by the same conjugacy written in the logarithmic chart). On the other hand, suppose that $f_{0}$ from (3.21) can be further reduced, or any of its coefficients changed, to, say, $f_{1}$. This would imply that, also in $z$-chart, $\tilde{f}$ can be normalized to $\tilde{f}_{1}(z):=\mathrm{e}^{-f_{1}(-\log z)}$. However, it is easy to check that $\tilde{f}_{1}$ and $\tilde{f}_{0}$ here defined differ in terms before the monomial $z \ell_{1} \ell_{2} \ell_{3} \ell_{4}^{1+\varepsilon}$, which is a contradiction with normalization result in [PRRS21], which states that terms before $z \ell_{1} \ell_{2} \ell_{3} \ell_{4}^{1+\varepsilon}$ in $\tilde{f}_{0}$ are not affected by formal changes of variables.
} 
From (3.22) and (3.23) and since $M_{\varepsilon, k}$ is decreasing on $[R,+\infty)$, we inductively obtain, for $n \in \mathbb{N}_{\geq 1}$ and $\zeta \in D_{R}^{f}$ :

$$
\begin{aligned}
\left|f^{\circ(n+1)}(\zeta)-(n+1) \beta-\left(f^{\circ n}(\zeta)-n \beta\right)\right| & =\left|f\left(f^{\circ n}(\zeta)\right)-\left(f^{\circ n}(\zeta)+\beta\right)\right| \\
& \leq M_{\varepsilon, k}\left(\Re\left(f^{\circ n}(\zeta)\right)\right) \\
& \leq M_{\varepsilon, k}\left(\Re(\zeta)+n \rho_{\beta, \varepsilon, k}^{-}(\Re(\zeta))\right) \\
& \leq M_{\varepsilon, k}\left(R+n \rho_{\beta, \varepsilon, k}^{-}(R)\right) .
\end{aligned}
$$

As stated in Subsection 3.1, the series $\sum_{n>0} M_{\varepsilon, k}\left(R+n \rho_{\beta, \varepsilon, k}^{-}(R)\right)$ converges. Therefore, the Koenigs sequence $\left(f^{\circ n}-n \beta\right)_{n}$ is uniformly Cauchy, hence converges uniformly on $D_{R}^{f}$. Denote by $\varphi$ its uniform limit on the domain $D_{R}^{f}$. By Weierstrass' theorem, it follows that $\varphi$ is analytic on $D_{R}^{f}$.

Finally, we compute:

$$
\begin{aligned}
(\varphi \circ f)(\zeta) & =\lim _{n}\left(f^{\circ n}(f(\zeta))-n \beta\right) \\
& =\lim _{n}\left(f^{\circ(n+1)}(\zeta)-(n+1) \beta\right)+\beta \\
& =\varphi(\zeta)+\beta .
\end{aligned}
$$

Therefore, $\varphi$ is an analytic linearization of $f$ on $D_{R}^{f}$, obtained as a uniform limit of the Koenigs sequence.

(ii) Suppose that $D_{R}^{f} \cap\{\zeta \in \mathbb{C}: \Im(\zeta)=0\}$ is invariant under $f(\zeta)=\zeta+\beta+o(1)$. Then obviously $\Im(\beta)=0$. Now consider the pointwise limit

$$
\varphi(\zeta):=\lim _{n \rightarrow \infty}\left(f^{\circ n}(\zeta)-n \beta\right), \zeta \in D_{R}^{f} \cap\{\zeta \in \mathbb{C}: \Im(\zeta)=0\} .
$$

Since $D_{R}^{f} \cap\left\{\zeta \in \mathbb{C}^{+}: \Im(\zeta)=0\right\}$ is invariant for (all iterates of) $f,\{\zeta \in \mathbb{C}: \Im(\zeta)=0\}$ closed in $\mathbb{C}^{+}$, and since $\Im(\beta)=0$, (3.25) implies that $\left\{D_{R}^{f} \cap\{\zeta \in \mathbb{C}: \Im(\zeta)=0\}\right.$ is invariant for $\varphi$.

(iii) For $0<\nu<\varepsilon, \zeta \in D_{R}^{f}$ and $m \in \mathbb{N}_{\geq 1}$, taking the sum of the terms

$$
f^{\circ(n+1)}(\zeta)-(n+1) \beta-\left(f^{\circ n}(\zeta)-n \beta\right)
$$

in (3.24) for $n$ ranging from 0 to $m-1$ it follows that

$$
\begin{aligned}
\left|f^{\circ(m)}(\zeta)-m \beta-\zeta\right| & \leq \sum_{n=0}^{m-1} M_{\varepsilon, k}\left(\Re(\zeta)+n \rho_{\beta, \varepsilon, k}^{-}(\Re(\zeta))\right) \\
& =\sum_{n=0}^{m-1} \frac{M_{\nu, k}\left(\Re(\zeta)+n \rho_{\beta, \varepsilon, k}^{-}(\Re(\zeta))\right)}{\left(\log ^{\circ k}\left(\Re(\zeta)+n \rho_{\beta, \varepsilon, k}^{-}(\Re(\zeta))\right)\right)^{\varepsilon-\nu}} \\
& \leq \frac{1}{\left(\log ^{\circ k}(\Re(\zeta))\right)^{\varepsilon-\nu}} \cdot \sum_{n=0}^{+\infty} M_{\nu, k}\left(R+n \rho_{\beta, \varepsilon, k}^{-}(R)\right) \\
& \leq \frac{C}{\left(\log ^{\circ k}(\Re(\zeta))\right)^{\varepsilon-\nu}}, C>0,
\end{aligned}
$$


where the last sum converges to $C>0$. Taking the pointwise limit for $m \rightarrow+\infty$ in (3.26), it follows that

$$
|\varphi(\zeta)-\zeta| \leq \frac{C}{\left(\log ^{\circ k}(\Re(\zeta))\right)^{\varepsilon-\nu}}, C>0, \zeta \in D_{R}^{f} .
$$

Therefore, $\varphi(\zeta)=\zeta+o(1)$, uniformly on $D_{R}^{f}$ as $\Re(\zeta) \rightarrow+\infty$.

To get a more rigorous estimate, using the elementary properties of the logarithm, we easily see that, for every domain $D_{h_{l}, h_{u}} \subseteq \mathbb{C}_{+}$such that $h_{l}(x)=O(x)$ and $h_{u}(x)=O(x)$, as $x \rightarrow+\infty$, there exists $N>0$, such that

$$
\left|\boldsymbol{L}_{k}\right| \leq N \log ^{\circ k}(\Re(\zeta)), \text { for } \zeta \in\left(D_{h_{l}, h_{u}}\right)_{R},
$$

for sufficiently large $R>\exp ^{\circ k}(0), k \in \mathbb{N}_{\geq 1}$. Indeed, the conditions $h_{l}(x)=O(x)$ and $h_{u}(x)=O(x)$ imply that there exists $d>0$ such that $|\Im(\zeta)| \leq d \cdot \Re(\zeta), \zeta \in$ $\left(D_{h_{l}, h_{u}}\right)_{R}$, for a sufficiently large $R>0$.

Using (3.28) in (3.27), it now follows that, for any $0<\nu<\nu^{\prime}<\varepsilon$, there exists $E>0$ such that:

$$
|\varphi(\zeta)-\zeta| \leq \frac{E}{\left(\log ^{\circ k}(\Re(\zeta))\right)^{\nu^{\prime}-\nu}}\left|\boldsymbol{L}_{k}\right|^{\nu^{\prime}-\varepsilon}, \zeta \in\left(D_{h_{l}, h_{u}}\right)_{R} .
$$

This implies that, for every $0<\nu^{\prime}<\varepsilon$, on $\left(D_{h_{l}, h_{u}}\right)_{R}$ it holds that:

$$
\lim _{\Re(\zeta) \rightarrow+\infty} \frac{\varphi(\zeta)-\zeta}{\frac{1}{\left(\boldsymbol{L}_{k}\right)^{\varepsilon-\nu^{\prime}}}}=0 .
$$

Therefore, for any $0<\nu<\varepsilon, \varphi(\zeta)=\zeta+o\left(\boldsymbol{L}_{k}^{-\nu}\right)$, as $\Re(\zeta) \rightarrow+\infty$ in $\left(D_{h_{l}, h_{u}}\right)_{R}$.

(iv) Suppose that $\psi$ is an analytic linearizing germ, i.e. $\psi \circ f=\psi+\beta$, on $f$ invariant subset $D_{1} \subseteq D$, such that $\psi(\zeta)=\zeta+o(1)$ uniformly on $D_{1}$ as $\Re(\zeta) \rightarrow+\infty$. Since $D_{1}$ is $f$-invariant and $D^{f}$ is a maximal $f$-invariant subdomain of $D$, obviously $\left(D_{1}\right)_{R} \subseteq D_{R}^{f}$. Clearly, $\left(D_{1}\right)_{R}$ is also $f$-invariant, and by (3.22), non-empty. Recall from (i) that $\varphi$ is the analytic linearization constructed on whole $D_{R}^{f}$ as the limit of the Koenigs sequence, for sufficiently large $R>\exp ^{\circ k}(0)$. It satisfies $\varphi(\zeta)=\zeta+o(1)$, uniformly as $\Re(\zeta) \rightarrow+\infty$ on $D_{R}^{f}$. We now show that $\psi \equiv \varphi$ on $\left(D_{1}\right)_{R}$.

Put

$$
E(\zeta):=\varphi(\zeta)-\psi(\zeta), \zeta \in\left(D_{1}\right)_{R}
$$

Then $E$ is analytic on $\left(D_{1}\right)_{R}$ and $E(\zeta)=o(1)$, as $\Re(\zeta) \rightarrow+\infty$ uniformly on $\left(D_{1}\right)_{R}$. Moreover, $(E \circ f)(\zeta)=E(\zeta), \zeta \in\left(D_{1}\right)_{R}$. Inductively, since $\left(D_{1}\right)_{R}$ is $f$-invariant, we obtain

$$
E\left(f^{\circ n}(\zeta)\right)=E(\zeta), \zeta \in\left(D_{1}\right)_{R}, n \in \mathbb{N} .
$$

By (3.22), $\Re\left(f^{\circ n}(\zeta)\right) \geq R+n \rho_{\beta, \varepsilon, k}^{-}(R)$, for $n \in \mathbb{N}$ and $\zeta \in\left(D_{1}\right)_{R} \subseteq D_{R}^{f}$. It follows that

$$
\lim _{n} \Re\left(f^{\circ n}(\zeta)\right)=+\infty, \zeta \in\left(D_{1}\right)_{R} .
$$

Passing to limit, as $n \rightarrow \infty$, in (3.30), and using (3.31) and the fact that $E(\zeta)=$ $o(1)$, as $\Re(\zeta) \rightarrow+\infty$, we get that $E(\zeta)=0$, for every $\zeta \in\left(D_{1}\right)_{R}$. That is, $\varphi \equiv \psi$ on $\left(D_{1}\right)_{R}$. 


\section{Linearization of Dulac maps}

This final section is dedicated to the proof of our main result (Theorem B, Section 2).

4.1. Linearization of a hyperbolic Dulac series. We prove in this subsection that a hyperbolic Dulac series (2.2) admits a unique (formal) parabolic Dulac linearization. A key argument in the proof is the linearization of hyperbolic logarithmic transseries established in [PRRS21]. Hence we first recall the notation used in [PRRS21]. Notice that, while the results there where established for logarithmic transseries with real coefficients, we need here to consider certain logarithmic transseries with complex coefficients. For the series considered here, as the proof consists of algebraic computations on the coefficients, switching from real to complex coefficients does not affect the results of [PRRS21].

Notation 4.1. In this section, $z$ denotes an infinitesimal variable and the symbol $\boldsymbol{\ell}_{1}$ denotes the transmonomial $-\frac{1}{\log z}$.

Following [PRRS21], we describe a class of logarithmic transseries in the variable $z$. We denote by $\mathcal{L}_{1}(\mathbb{C})$ the collection of logarithmic transseries of the form

$$
\widehat{f_{1}}=\sum_{(\alpha, n) \in \mathbb{R}_{\geq 0} \times \mathbb{Z}} a_{\alpha, n} \cdot z^{\alpha} \ell_{1}^{n},
$$

where $\operatorname{Supp}\left(\widehat{f}_{1}\right):=\left\{(\alpha, n) \in \mathbb{R}_{\geq 0} \times \mathbb{Z}: a_{\alpha, n} \neq 0\right\}$, called the support of $\widehat{f}_{1}$, is a well-ordered subset of $\mathbb{R}_{\geq 0} \times \mathbb{Z}$ (for the lexicographic order), and $a_{\alpha, n} \in \mathbb{C}$.

For $\widehat{f}_{1} \in \mathcal{L}_{1}(\mathbb{C})$, let ord $\left(\widehat{f}_{1}\right):=\min \operatorname{Supp}\left(\widehat{f}_{1}\right) \in \mathbb{R}_{\geq 0} \times \mathbb{Z}$ if $\widehat{f}_{1} \neq 0$ and $\operatorname{ord}(0)=+\infty$. Let $\operatorname{ord}_{z}\left(\widehat{f}_{1}\right)$ be the smallest $\alpha \in \mathbb{R}_{\geq 0}$ such that there exists $n \in \mathbb{Z}$ with $(\alpha, n) \in \operatorname{Supp}\left(\widehat{f}_{1}\right)$ if $\widehat{f}_{1} \neq 0$ and $\operatorname{ord}_{z}(0)=+\infty$. We also use the acronym h.o.t. for "higher order terms" when we describe a transseries.

Consider a transseries $\widehat{f}_{1} \in \mathcal{L}_{1}(\mathbb{C})$ such that ord $\left(\widehat{f}_{1}\right)=(1,0)$. If $a_{1,0}=1$, the transseries $\widehat{f_{1}}$ is called parabolic. If $\left|a_{1,0}\right| \neq 0,1$, the series $\widehat{f}_{1}$ is called hyperbolic.

An element of $\mathcal{L}_{1}(\mathbb{C})$ can also be written blockwise:

$$
\widehat{f_{1}}=\sum_{\alpha \in \mathbb{R}_{\geq 0}} z^{\alpha} R_{\alpha}\left(\boldsymbol{\ell}_{1}\right)
$$

where each $R_{\alpha}$, called the block of index $\alpha$, is an element of the field of Laurent series $\mathbb{C}\left(\left(\boldsymbol{\ell}_{1}\right)\right)$.

To a parabolic transseries $\widehat{f}_{1}(z)$ as in (4.2) corresponds the parabolic complex exponential transseries in the infinite variable $\zeta=-\log z$

$$
\widehat{f}(\zeta)=-\log \left(\widehat{f}_{1}(\exp (-\zeta))\right)=\zeta+\sum_{\nu \geq 0} \exp (-\nu \zeta) P_{\nu}\left(\zeta^{-1}\right)
$$

where, for each $\nu, P_{\nu} \in \mathbb{C}((X))$ is a Laurent series, and $\varepsilon(\zeta):=\widehat{f}(\zeta)-\zeta$ is an infinitesimal transseries.

A transseries in $\mathcal{L}_{1}(\mathbb{C})$ might fail to be a logarithmic Dulac series (2.4) for two reasons: 
1. The exponents $\alpha$ form a well-ordered subset of $\mathbb{R}_{>0}$, but in general they do not belong to a finitely generated sub-semigroup of $\mathbb{R}_{>0}$. Moreover, they might not form a strictly increasing sequence tending to $+\infty$.

2. The blocks $R_{\alpha}$ are Laurent series in $\ell_{1}$, but they are not necessarily complex polynomials in $\ell_{1}^{-1}$ (additionally, in a Dulac series, the polynomial in the leading block must necessarily be a constant).

These are precisely the two properties that have to be checked to guarantee that an element of $\mathcal{L}_{1}(\mathbb{C})$ is a complex Dulac series, as in the proof of the following lemma.

Lemma 4.2 (Formal linearization). Let $\widehat{f}=\zeta+\beta+$ h.o.t., $\beta \in \mathbb{C}^{+}$, be a hyperbolic complex Dulac series (2.4). Then there exists a unique parabolic complex exponential transseries $\widehat{\varphi}$ such that $\widehat{\varphi} \circ \widehat{f}=\widehat{\varphi}+\beta$. Moreover, $\widehat{\varphi}$ is a complex Dulac transseries as in (2.2). Finally, if the coefficients of $\widehat{f}$ are real, then so are those of $\widehat{\varphi}$.

Proof. Existence. Let $\lambda:=\exp (-\beta) \in \mathbb{C}$, where exp is the complex exponential function and not the compositional inverse of the logarithmic chart. Let

$$
\begin{aligned}
\widehat{f}_{1}(z) & :=\exp (-\widehat{f}(-\log z)) \\
& =\exp \left(-\left(-\log z+\beta+\sum_{i=1}^{\infty} \exp \left(\nu_{i} \log z\right) R_{i}(-\log z)\right)\right), R_{i} \in \mathbb{C}[X], \nu_{i}>0, \\
& =\lambda z \exp \left(\sum_{i=1}^{\infty} z^{\nu_{i}} R_{i}(-\log z)\right) \\
& =\lambda z+\sum_{i=1}^{\infty} z^{\alpha_{i}} P_{i}(\log z), \quad \alpha_{i}>1, P_{i} \in \mathbb{C}[X],
\end{aligned}
$$

so that $\widehat{f}_{1} \in \mathcal{L}_{1}(\mathbb{C})$ is a complex logarithmic Dulac series in the variable $z$. Notice that here $\lambda$ is a complex number, and is not seen as the element of $\widetilde{\mathbb{C}}$ parameterized by $\beta$ in the logarithmic chart. It is indeed important that all the coefficients of $\widehat{f}_{1}$ are complex numbers and not elements of $\widetilde{\mathbb{C}}$, in order to apply to them all the algebraic computations involved in the proof of the Main Theorem of [PRRS21].

The latter implies that $\widehat{f}_{1}$ admits a unique parabolic linearization $\widehat{\varphi}_{1} \in \mathcal{L}_{1}(\mathbb{C})$. Let $\widehat{g}_{1}:=\widehat{f}_{1}-\lambda$. id and $\gamma:=\operatorname{ord}_{z}\left(\widehat{g}_{1}\right)$. As $\widehat{f}_{1}$ is a Dulac series, we have $\gamma>1$. Moreover, recall that the exponents of $z$ in $\widehat{f}_{1}$ form a finitely generated strictly positive sequence which tends to $+\infty$. Hence, we deduce from the description of the support of $\widehat{\varphi}_{1}$ given in [PRRS21, Section 5] that the exponents of $z$ in $\widehat{\varphi}_{1}$ also form a finitely generated strictly positive sequence which tends to $+\infty$.

We now prove the polynomial property for blocks of the linearization $\widehat{\varphi}_{1}$ : that each monomial $z^{\alpha}$ in $\widehat{\varphi}_{1}$ is multiplied by a complex polynomial in $\ell_{1}^{-1}=-\log z$. By the proof of the Main Theorem in [PRRS21], the linearization $\widehat{\varphi}_{1}$ is given by

$$
\widehat{\varphi}_{1}:=\mathrm{id}+\widehat{h}_{1},
$$

where $\widehat{h}_{1} \in \mathcal{L}_{1}(\mathbb{C}), \operatorname{ord}_{z}\left(\widehat{h}_{1}\right)>1$, is the limit of the Picard sequence $\left(\widehat{\psi}_{n}\right)_{n \in \mathbb{N}}$ defined by

$$
\widehat{\psi}_{n}:=\left(\mathcal{T}_{\widehat{f}_{1}}^{-1} \circ \mathcal{S}_{\widehat{f}_{1}}\right)^{\circ n}(0)
$$


Here, the limit is taken in the sense of the valuation topology (see e.g. [DMM01]): $\operatorname{ord}_{z}\left(\widehat{\psi}_{n}-\widehat{\varphi}_{1}\right)$ tends to $+\infty$, as $n \rightarrow+\infty$. The operators $\mathcal{T}_{\widehat{f}_{1}}$ and $\mathcal{S}_{\widehat{f}_{1}}(\operatorname{case} \beta>1)$ are defined in [PRRS21] as

$$
\mathcal{S}_{\widehat{f}_{1}}(\widehat{h})=\frac{1}{\lambda} \widehat{g}_{1}+\frac{1}{\lambda} \sum_{i \geq 1} \frac{\widehat{h}^{(i)}(\lambda z)}{i !} \widehat{g}_{1}^{i}, \text { and } \mathcal{T}_{\widehat{f}_{1}}(\widehat{h})=\widehat{h}-\frac{1}{\lambda} \widehat{h}(\lambda z) .
$$

In (4.5), transseries are applied to $\lambda z$. This means, using the following compositional rules, that

$$
\log (\lambda z)=\log (\lambda)+\log (z)=-\beta+\log (z)
$$

and, for $\alpha>0$,

$$
(\lambda z)^{\alpha}=\lambda^{\alpha} z^{\alpha}=\exp (\alpha \log (\lambda)) z^{\alpha}=\exp (-\alpha \beta) z^{\alpha} .
$$

In particular, we see that, in this proof, $\lambda$ is the only complex number for which we have to impose a determination of the logarithm. We chose $\log (\lambda)=-\beta$ in view of the final step of the proof, in which we deduce the linearization of $\widehat{f}$ from the linearization of $\widehat{f}_{1}$.

Now, due to the convergence of (4.4) to the linearization $\widehat{\varphi}_{1}$ in the valuation topology, it suffices to prove the following: if $\widehat{h} \in \mathcal{L}_{1}(\mathbb{C})$ with $\operatorname{ord}_{z}(\widehat{h})>1$ satisfies the polynomial property, the same holds for $\mathcal{T}_{\widehat{f}_{1}}^{-1}(\widehat{h})$ and for $\mathcal{S}_{\widehat{f}_{1}}(\widehat{h})$.

Notice that the polynomial property is preserved under differentiation, under multiplication by a complex Dulac series, as well as under precomposition with $\lambda z$. Therefore, if $\widehat{h}$ has the polynomial property, then so does $\mathcal{S}_{\widehat{f}_{1}}(\widehat{h})$, thanks to (4.5), the previous remark and the fact that, as $\gamma>1, \mathcal{S}_{\widehat{f}_{1}}$ is an infinite sum of operators which strictly increase $\operatorname{ord}_{z}$.

Let us now check the polynomial property for $\mathcal{T}_{\widehat{f}_{1}}^{-1}(\widehat{h})$. Suppose the contrary, that is, that there exists $\widehat{h} \in \mathcal{L}_{1}(\mathbb{C}) \operatorname{ord}_{z}(\widehat{h})>1$, which satisfies the polynomial property, while $\mathcal{T}_{\widehat{f}_{1}}^{-1}(\widehat{h})$ does not. Then $\mathcal{T}_{\widehat{f}_{1}}^{-1}(\widehat{h})$ admits a block $\widehat{R}_{\nu}\left(\boldsymbol{\ell}_{1}\right) \in \mathbb{C}\left(\left(\boldsymbol{\ell}_{1}\right)\right)$, for some $\nu>1$, which is not a polynomial in $\ell_{1}^{-1}$. Hence we can write

$$
\widehat{R}_{\nu}\left(\ell_{1}\right)=Q\left(\ell_{1}^{-1}\right)+a\left(\ell_{1}\right), \text { with } a\left(\ell_{1}\right)=\sum_{n \geq n_{0}} a_{n} \ell_{1}^{n}, a_{n} \in \mathbb{C},
$$

where $Q \in \mathbb{C}\left[\ell_{1}^{-1}\right]$ is a polynomial and $a \in \mathbb{C}\left[\left[\ell_{1}\right]\right]$ is a nonzero power series such that $a_{n_{0}} \neq 0, n_{0} \in \mathbb{N}_{\geq 1}$. Now apply $\mathcal{T}_{\widehat{f}_{1}}$ from $(4.5)$ to such $\mathcal{T}_{\widehat{f}_{1}}^{-1}(\widehat{h})$, to obtain $\widehat{h}$. However, using (4.5) and the evident relations (see [PRRS21, Section 3.6])

$$
\begin{aligned}
& \boldsymbol{\ell}_{1}^{-1}(\lambda z)=\boldsymbol{\ell}_{1}^{-1}-\log \lambda=\boldsymbol{\ell}_{1}^{-1}+\beta, \\
& \boldsymbol{\ell}_{1}(\lambda z)=\boldsymbol{\ell}_{1} \cdot\left(1+\varepsilon\left(\boldsymbol{\ell}_{1}\right)\right), \text { for some } \varepsilon \in \mathbb{C}\left[\left[\boldsymbol{\ell}_{1}\right]\right], \varepsilon(0)=0, \\
& \boldsymbol{\ell}_{1}^{n}(\lambda z)=\boldsymbol{\ell}_{1}^{n} \cdot\left(1+\varepsilon_{n}\left(\boldsymbol{\ell}_{1}\right)\right), \text { for some } \varepsilon_{n} \in \mathbb{C}\left[\left[\boldsymbol{\ell}_{1}\right]\right], \varepsilon_{n}(0)=0\left(n \in \mathbb{N}_{\geq 1}\right),
\end{aligned}
$$

it is easy to see that the block of index $\nu$ in $\widehat{h}=\mathcal{T}_{\widehat{f}_{1}}\left(\mathcal{T}_{\widehat{f}_{1}}^{-1}(\widehat{h})\right)$ is

$$
Q\left(\ell_{1}^{-1}\right)+a\left(\ell_{1}\right)-\lambda^{\nu-1}\left(Q\left(\ell_{1}^{-1}+\beta\right)+\widetilde{a}\left(\ell_{1}\right)\right),
$$

where $\widetilde{a} \in \mathbb{C}\left[\left[\ell_{1}\right]\right]$ is a power series such that $\widetilde{a}(0)=0$ and with $a_{n_{0}} \ell_{1}^{n_{0}}$ as leading term. The power series $a-\lambda^{\nu-1} \widetilde{a} \in \mathbb{C}\left[\left[\ell_{1}\right]\right]$ does not have a constant term, but is 
nonzero because its smallest coefficient is equal to $\left(1-\lambda^{\nu-1}\right) a_{n_{0}} \neq 0$. This contradicts the fact that the block of index $\nu$ of $\widehat{h}$ is a polynomial in $\ell_{1}^{-1}$. Therefore, $\widehat{\varphi}_{1}$ is a logarithmic Dulac series.

Finally, let $\widehat{\varphi}(\zeta):=-\log \left(\widehat{\varphi}_{1}(\exp (-\zeta))\right)$. Since we chose $\log (\lambda)=-\beta$, we have that $\widehat{f}(\zeta)=-\log \left(\widehat{f}_{1}(\exp (-\zeta))\right)$ and $-\log \left(\lambda \widehat{\varphi}_{1}(\exp (-\zeta))\right)=\widehat{\varphi}(\zeta)+\beta$. Hence we deduce from $\widehat{\varphi}_{1} \circ \widehat{f}_{1}=\lambda \widehat{\varphi}_{1}$ that $\widehat{\varphi} \circ \widehat{f}=\widehat{\varphi}+\beta$.

Notice that in this proof, if the coefficients of $\widehat{f}$ are real, so are the coefficients of $\widehat{f}_{1}, \widehat{\varphi}_{1}$, and $\widehat{\varphi}$.

Uniqueness. We have $\widehat{f}(\zeta)=\zeta+\beta+\widehat{\varepsilon}(\zeta)$, where $\widehat{\varepsilon}(\zeta)$ is an infinitesimal complex exponential transeries with the polynomial property. The difference $\widehat{\psi}$ between two parabolic complex linearizations of $\widehat{f}$ of type (4.3) satisfies

$$
\widehat{\psi} \circ \widehat{f}=\widehat{\psi} \text {. }
$$

If $\widehat{\psi} \neq 0$, then its leading term is $a \zeta^{p} \exp (-\nu \zeta)$ for some $a \in \mathbb{C} \backslash\{0\}, \nu \geq 0$ and $p \in \mathbb{Z}$. There are two cases.

If $\nu>0$, then the leading term of $\widehat{\psi} \circ \widehat{f}$ is $a \zeta^{p} \exp (-\nu \zeta) \exp (-\nu \beta)$ (because $\exp (\varepsilon(\zeta))=1+\eta(\zeta)$, where $\eta$ is an infinitesimal transseries). It follows from (4.7) that $\exp (-\nu \beta)=1$, which is impossible, as $\Re(\beta)>0$ and $\nu>0$.

If $\nu=0$, then $p<0$. In that case, $\widehat{\psi}(\zeta)=a \zeta^{p}+b \zeta^{p-1}+$ h.o.t., with $b \in \mathbb{C}$ possibly equal to 0 . Comparing the coefficients of $\zeta^{p-1}$ on both sides of (4.7), we see that $p a \beta$ must be 0 , a contradiction.

4.2. Partial linearizations and homological equations. Consider a nontrivial hyperbolic complex Dulac map $f$ on a standard quadratic domain $\mathcal{R}_{C} \subset \mathbb{C}^{+}$ (nontrivial meaning that $f(\zeta) \neq \zeta+\beta, \beta \in \mathbb{C}^{+}$). Then $f$ admits a nontrivial (by quasianalyticity) complex Dulac expansion $\widehat{f}=\zeta+\beta+\exp \left(-\alpha_{1} \zeta\right) P_{1}(\zeta)+$ h.o.t., where $\alpha_{1}>0$ and $P_{1} \in \mathbb{C}[\zeta]$. Hence $f$ satisfies the hypotheses of Theorem $\mathrm{A}$ in Section 3. Therefore, by Theorem A, $f$ admits an analytic linearization on the invariant domain $\left(\mathcal{R}_{C}\right)_{R}^{f}$, for $R>0$ sufficiently large.

Note that here $\left(\mathcal{R}_{C}\right)_{R}^{f}=\left(\mathcal{R}_{C}\right)_{R}$, for $R>0$ sufficiently large. Indeed, by Example (3), it follows that if $\mathcal{R}_{C}$ is a standard quadratic domain, then for a sufficiently large $R>0,\left(\mathcal{R}_{C}\right)_{R}$ is a domain of type $(\beta, \varepsilon, k), \varepsilon>0$ and $k \in \mathbb{N}_{\geq 1}$. Proposition 3.4 implies that $\left(\mathcal{R}_{C}\right)_{R}$, for a sufficiently large $R>0$, is $f$-invariant. Therefore, it is easy to see that $\left(\mathcal{R}_{C}\right)_{R}^{f}=\left(\mathcal{R}_{C}\right)_{R}$, for a sufficiently large $R>0$. Now let $D:=\left(\mathcal{R}_{C}\right)_{R}$. By Definition 2.1, we call such a domain $D$ a germ of the standard quadratic domain $\mathcal{R}_{C}$, since, as a germ, it is equal to $\mathcal{R}_{C}$.

By Theorem $\mathrm{A}$, there exists an analytic linearization $\varphi$ of $f$ on $D$, which is unique in the class of tangent to the identity linearizations on $f$-invariant subdomains. On the other hand, on the formal side, we apply the formal linearization Lemma 4.2 to $\widehat{f}$, to obtain the unique formal parabolic Dulac linearization $\widehat{\varphi}$. We say that $f$ is formally linearizable (by a parabolic complex Dulac series).

In the sequel, we prove the lemmas which will be used in the proof of Theorem B in Subsection 4.3, to show that the formal linearization $\widehat{\varphi}$ is the Dulac asymptotic 
expansion of the analytic linearization $\varphi$ on some standard quadratic subdomain of $D$

The following Lemma 4.3 shows how the partial sums of the formal linearization of a hyperbolic complex Dulac map perform its approximate analytic linearizations on its domain. Such partial linearizations satisfy the homological equations (4.10).

Lemma 4.3 (Partial linearizations). Let $f(\zeta)=\zeta+\beta+o(1), \beta \in \mathbb{C}^{+}$, be a nontrivial hyperbolic complex Dulac map defined on a standard quadratic domain $\mathcal{R}_{C}$. Let the parabolic Dulac series

$$
\widehat{\varphi}(\zeta)=\zeta+\sum_{i=1}^{\infty} \mathrm{e}^{-\beta_{i} \zeta} Q_{i}(\zeta)
$$

where $Q_{i} \in \mathbb{C}[\zeta]$ and $\left(\beta_{i}\right)_{i}$ is a strictly increasing sequence of positive real numbers tending to $+\infty$, be its formal linearization from Lemma 4.2. Here, if $\widehat{\varphi}$ is a finite sum, that is, if there exists $i_{0} \in \mathbb{N}$ such that $Q_{i}=0$ for $i>i_{0}$, we take any strictly increasing sequence $\left(\beta_{i}\right)_{i>i_{0}}$ such that $\beta_{i}>\beta_{i_{0}}$ and $\beta_{i} \rightarrow+\infty$. Let

$$
\begin{aligned}
& \widehat{\varphi}_{0}:=\zeta, \\
& \widehat{\varphi}_{n}:=\zeta+\sum_{i=1}^{n} \mathrm{e}^{-\beta_{i} \zeta} Q_{i}(\zeta), n \in \mathbb{N}_{\geq 1},
\end{aligned}
$$

be the partial sums of $\widehat{\varphi}$, and $\varphi_{n}$ be the analytic germs on $\mathbb{C}^{+}$defined by the finite sums $\widehat{\varphi}_{n}, n \in \mathbb{N}$. Then, for every $n \in \mathbb{N}$, there exists $\nu_{n}>0$, such that

$$
\left(\varphi_{n} \circ f\right)(\zeta)-\varphi_{n}(\zeta)=\beta+o\left(\mathrm{e}^{-\left(\beta_{n}+\nu_{n}\right) \zeta}\right)
$$

uniformly on $\mathcal{R}_{C}$ as $\Re(\zeta) \rightarrow+\infty$. Here, $\beta_{0}=0$.

Note that, if $\widehat{\varphi}$ is a finite sum, the sequence $\left(\varphi_{n}\right)_{n \in \mathbb{N}}$ eventually stabilizes.

Proof. Let

$$
\widehat{f}(\zeta)=\zeta+\beta+\sum_{i=1}^{\infty} \mathrm{e}^{-\alpha_{i} \zeta} P_{i}(\zeta), P_{i} \in \mathbb{C}[\zeta], i \in \mathbb{N}_{\geq 1},
$$

where $\left(\alpha_{i}\right)_{i}$ is a strictly increasing sequence of strictly positive real numbers tending to $+\infty$, be the (complex) Dulac expansion of $f$. Recall that this expansion of $f$ is uniform on a standard quadratic domain $\mathcal{R}_{C}$, as $\Re(\zeta) \rightarrow+\infty$. For $n \in \mathbb{N}$, let

$$
\begin{aligned}
& \widehat{f}_{0}:=\zeta+\beta, \\
& \widehat{f}_{n}:=\zeta+\beta+\sum_{i \in \mathbb{N}_{\geq 1}: \alpha_{i} \leq \beta_{n}} \mathrm{e}^{-\alpha_{i} \zeta} P_{i}(\zeta), \quad n \in \mathbb{N}_{\geq 1},
\end{aligned}
$$

be the partial sums of $\widehat{f}$. Furthermore, let $\widehat{g}_{n}:=\widehat{f}-\widehat{f}_{n}$, for $n \in \mathbb{N}$. The composition $\widehat{\varphi} \circ \widehat{f}$ can be computed as

$$
\widehat{\varphi} \circ \widehat{f}=\widehat{\varphi}\left(\widehat{f}_{n}+\widehat{g}_{n}\right)=\widehat{\varphi} \circ \widehat{f}_{n}+\sum_{i \geq 1} \frac{\widehat{\varphi}^{(i)} \circ \widehat{f}_{n}}{i !} \widehat{g}_{n}^{i},
$$

as the series in (4.11) converges for the valuation topology. Obviously,

$$
\widehat{\varphi} \circ \widehat{f}_{n}=\widehat{\varphi}_{n} \circ \widehat{f}_{n}+\left(\widehat{\varphi}-\widehat{\varphi}_{n}\right) \circ \widehat{f}_{n}, \quad n \in \mathbb{N} .
$$


Now, using (4.11) and (4.12) and the fact that $\widehat{\varphi}$ is the formal linearization of $\widehat{f}$, we get:

$$
\begin{aligned}
0 & =\widehat{\varphi} \circ \widehat{f}-\widehat{\varphi}-\beta \\
& =\widehat{\varphi}_{n} \circ \widehat{f}_{n}-\widehat{\varphi}_{n}+\left(\widehat{\varphi}-\widehat{\varphi}_{n}\right) \circ \widehat{f}_{n}-\left(\widehat{\varphi}-\widehat{\varphi}_{n}\right)+\sum_{i \geq 1} \frac{\widehat{\varphi}^{(i)} \circ \widehat{f}_{n}}{i !} \widehat{g}_{n}^{i}-\beta,
\end{aligned}
$$

for $n \in \mathbb{N}$. For every $n \in \mathbb{N}$, it can easily be seen that there exists $\mu_{n}>0$, such that:

$$
\operatorname{ord}_{\mathrm{e}^{-\zeta}}\left(\left(\widehat{\varphi}-\widehat{\varphi}_{n}\right) \circ \widehat{f}_{n}-\left(\widehat{\varphi}-\widehat{\varphi}_{n}\right)+\sum_{i \geq 1} \frac{\widehat{\varphi}^{(i)} \circ \widehat{f}_{n}}{i !} \widehat{g}_{n}^{i}\right)>\beta_{n}+\mu_{n} .
$$

From (4.13) and (4.14), we obtain that

$$
\operatorname{ord}_{\mathrm{e}^{-\zeta}}\left(\widehat{\varphi}_{n} \circ \widehat{f}_{n}-\widehat{\varphi}_{n}-\beta\right)>\beta_{n}+\mu_{n}, \quad n \in \mathbb{N} .
$$

As the sums in $\widehat{\varphi}_{n}$ and $\widehat{f}_{n}$ are finite, they define analytic germs $\varphi_{n}$ and $f_{n}$ on $\mathbb{C}^{+}$, in the $\zeta$-chart. This implies that

$$
\varphi_{n} \circ f_{n}-\varphi_{n}-\beta=o\left(\mathrm{e}^{-\left(\beta_{n}+\mu_{n}\right) \zeta}\right), \Re(\zeta) \rightarrow+\infty \text { on } \mathbb{C}^{+},
$$

for $n \in \mathbb{N}$. Moreover, due to the fact that $f_{n}$ and $\varphi_{n}, n \in \mathbb{N}$, are finite sums of power-exponential monomials, the convergence is uniform if we restrict to the standard quadratic domain $\mathcal{R}_{C}$ (since the imaginary part is bounded by a power of the real part along this domain).

Now put $g_{n}(\zeta):=f(\zeta)-f_{n}(\zeta)$, for $\zeta \in \mathcal{R}_{C}$ and $n \in \mathbb{N}$. It is obvious that $g_{n} \sim \widehat{g}_{n}$ uniformly on $\mathcal{R}_{C}$ as $\Re(\zeta) \rightarrow+\infty$. By Taylor's Theorem, (4.16), and since $\varphi_{n}$ is a finite sum of monomials with uniform asymptotics on $\mathcal{R}_{C}$, it follows that for every $n \in \mathbb{N}$ there exists some $\nu_{n}>0$ such that

$$
\begin{aligned}
\varphi_{n} \circ f-\varphi_{n}-\beta & =\varphi_{n}\left(f_{n}+g_{n}\right)-\varphi_{n}-\beta \\
& =\varphi_{n} \circ f_{n}-\varphi_{n}-\beta+\sum_{i=1}^{+\infty} \frac{\varphi_{n}^{(i)}\left(f_{n}\right)}{i !} g_{n}^{i} \\
& =o\left(\mathrm{e}^{-\left(\beta_{n}+\nu_{n}\right) \zeta}\right),
\end{aligned}
$$

uniformly on the standard quadratic domain $\mathcal{R}_{C}$, as $\Re(\zeta) \rightarrow+\infty$.

Lemma 4.4 (below) shows how to solve and give an estimate of the solution of a particular homological equation, that resembles the Abel's equation. The idea of the proof is taken from [Lor98]. This will be used in the proof of Theorem B (in Subsection 4.3) to control the growth of the differences between the analytic linearization of a hyperbolic complex Dulac map, given by Theorem A, and its partial linearizations by truncated complex Dulac sums given by (4.9) in Lemma 4.3. These differences themselves solve particular homological equations.

This estimates allow us to conclude, in the proof of Theorem B in Subsection 4.3, that a hyperbolic complex Dulac germ admits a parabolic complex Dulac linearization on some invariant standard quadratic (sub)domain of its domain of definition. 
Lemma 4.4 (Explicit analytic solutions to Abel-type homological equations). Let $f$ be a hyperbolic complex Dulac germ defined on a standard quadratic domain $\mathcal{R}_{C} \subset \mathbb{C}^{+}$. Let $h$ be an analytic map on $\mathcal{R}_{C}$, such that $h(\zeta)=o\left(\mathrm{e}^{-\alpha \zeta}\right)$ for some $\alpha>0$, uniformly on $\mathcal{R}_{C}$ as $\Re(\zeta) \rightarrow+\infty$. Then:

1. (Existence of an analytic solution to a homological equation) There exist $R>0$ such that $D:=\left(\mathcal{R}_{C}\right)_{R}$ is an $f$-invariant subdomain $D \subseteq \mathcal{R}_{C}$, and an analytic solution $\psi$ of the homological equation

$$
(\psi \circ f)(\zeta)-\psi(\zeta)=h(\zeta)
$$

on the subdomain $D$.

2. (Estimate of the solution) The following estimate holds:

$$
\psi(\zeta)=O\left(\mathrm{e}^{-\alpha \zeta}\right)
$$

uniformly on $D$ as $\Re(\zeta) \rightarrow+\infty$.

3. (Uniqueness of the solution) If $\psi_{1}$ is an analytic solution of equation (4.17) on an $f$-invariant subdomain $D_{1} \subseteq \mathcal{R}_{C}$, such that $\psi_{1}(\zeta)=o(1)$ uniformly on $D_{1}$ as $\Re(\zeta) \rightarrow+\infty$, then

$$
\psi_{1} \equiv \psi \text { on }\left(D_{1}\right)_{R}=D \cap D_{1} .
$$

Proof.

1. Existence of a solution. We prove that the following series:

$$
\psi(\zeta):=-\sum_{n=0}^{+\infty} h\left(f^{\circ n}(\zeta)\right)
$$

converges uniformly on $D$ (in the $\zeta$-chart) to an analytic map $\psi$ which satisfies equation (4.17).

Since $h(\zeta)=o\left(\mathrm{e}^{-\alpha \zeta}\right)$ uniformly on $\mathcal{R}_{C}$ as $\Re(\zeta) \rightarrow+\infty$, there exists $R>0$ such that

$$
|h(\zeta)| \leq\left|\mathrm{e}^{-\alpha \zeta}\right|=\frac{1}{\mathrm{e}^{\alpha \Re(\zeta)}} \leq \frac{1}{\mathrm{e}^{\alpha R}}
$$

for $\zeta \in \mathcal{R}_{C}, \Re(\zeta) \geq R$.

Let $\varepsilon>0$ and $k \in \mathbb{N}$ be arbitrary. By the discussion at the beginning of Subsection 4.2, we take $R>0$ sufficiently large such that $\left(\mathcal{R}_{C}\right)_{R}=\left(\mathcal{R}_{C}\right)_{R}^{f}$, that is, such that the whole of $\left(\mathcal{R}_{C}\right)_{R}$ is $f$-invariant. Now, put $D:=\left(\mathcal{R}_{C}\right)_{R}$. From (3.22) it follows that

$$
\Re\left(f^{\circ n}(\zeta)\right) \geq \Re(\zeta)+n \rho_{\beta, \varepsilon, k}^{-}(R) \geq R+n \rho_{\beta, \varepsilon, k}^{-}(R), \text { for } \zeta \in D,
$$

for $n \in \mathbb{N}$. Now, from (4.20) and (4.21), it follows that, for $n \in \mathbb{N}$ and $\zeta \in D$

$$
\left|h\left(f^{\circ n}(\zeta)\right)\right| \leq \frac{1}{\mathrm{e}^{\alpha \Re\left(f^{\circ n}(\zeta)\right)}} \leq \frac{1}{\mathrm{e}^{\alpha R}} \cdot\left(\frac{1}{\mathrm{e}^{\alpha \cdot \rho_{\beta, \varepsilon, k}^{-}(R)}}\right)^{n} .
$$


This implies that sum (4.19) converges uniformly on $D$. By Weierstrass' Theorem, it follows that $\psi$ defined by (4.19) is analytic on $D$. Now (4.17) follows easily:

$$
\begin{aligned}
\psi(f(\zeta)) & =-\sum_{n=0}^{+\infty} h\left(f^{\circ(n+1)}(\zeta)\right) \\
& =-\sum_{n=1}^{+\infty} h\left(f^{\circ n}(\zeta)\right) \\
& =-(-\psi(\zeta)-h(\zeta)) \\
& =\psi(\zeta)+h(\zeta), \text { for } \zeta \in D .
\end{aligned}
$$

2. Asymptotics of the solution $\psi$. From (4.19), (4.20) and (4.21) it follows that

$$
|\psi(\zeta)| \leq \sum_{n=0}^{\infty}\left|h\left(f^{\circ n}(\zeta)\right)\right| \leq \mathrm{e}^{-\alpha \Re(\zeta)} \cdot \frac{1}{1-\frac{1}{\mathrm{e}^{\alpha \cdot \rho_{\beta, \varepsilon, k}(R)}}},
$$

for $\zeta \in D$. This implies that $\psi(\zeta)=O\left(\mathrm{e}^{-\alpha \zeta}\right)$ uniformly on $D$ as $\Re(\zeta) \rightarrow+\infty$.

3. Uniqueness of the solution. Suppose that there exists an analytic solution $\psi_{1}$ to the homological equation (4.17), defined on an $f$-invariant subdomain $D_{1} \subseteq \mathcal{R}_{C}$, such that $\psi_{1}(\zeta)=o(1)$ uniformly on $D_{1}$ as $\Re(\zeta) \rightarrow+\infty$. By (4.21), note that $\left(D_{1}\right)_{R}=D_{1} \cap D$ is a nonempty $f$-invariant subdomain of $D$. Let

$$
\psi_{2}(\zeta):=\psi(\zeta)-\psi_{1}(\zeta), \zeta \in\left(D_{1}\right)_{R} .
$$

Since both $\psi$ and $\psi_{1}$ satisfy equation (4.17) on $\left(D_{1}\right)_{R}$ and $\psi(\zeta)=o(1), \psi_{1}(\zeta)=o(1)$, we have that $\psi_{2}(f(\zeta))=\psi_{2}(\zeta)$, for $\zeta \in\left(D_{1}\right)_{R}$, and $\psi_{2}(\zeta)=o(1)$ uniformly on $\left(D_{1}\right)_{R}$ as $\Re(\zeta) \rightarrow+\infty$. Therefore,

$$
\psi_{2}\left(f^{\circ n}(\zeta)\right)=\psi_{2}(\zeta), \zeta \in\left(D_{1}\right)_{R}, n \in \mathbb{N} .
$$

Note that $\psi_{2}(\zeta)=o(1)$, as $\Re(\zeta) \rightarrow+\infty$ uniformly on $\left(D_{1}\right)_{R}$. From (4.21) it follows that $\Re\left(f^{\circ n}(\zeta)\right) \rightarrow+\infty$ as $n \rightarrow \infty$, for every $\zeta \in\left(D_{1}\right)_{R}$. Therefore, passing to the limit as $n \rightarrow \infty$ in (4.22), we obtain that $\psi_{2} \equiv 0$ on $\left(D_{1}\right)_{R}$. Therefore, $\psi \equiv \psi_{1}$ on $\left(D_{1}\right)_{R}$.

\subsection{Proof of Theorem B: uniqueness and existence of a (complex) Dulac linearization.}

We now gather all the previous results to finish the proof of Theorem B.

Proof of Theorem B. Let $f(\zeta)=\zeta+\beta+o(1)$, for $\beta \in \mathbb{C}^{+}$, be a hyperbolic complex Dulac germ on a standard quadratic domain $\mathcal{R}_{C}$ and let $\widehat{f}$ be its complex Dulac expansion.

If $\widehat{f}=\zeta+\beta$, then, by quasianalyticity, it follows that $f(\zeta)=\zeta+\beta$ for $\zeta \in \mathcal{R}_{C}$, so that $f$ is already linearized.

Now, suppose that both $f$ and $\widehat{f}$ is nontrivial. By Lemma 4.2, there exists a unique formal linearization $\widehat{\varphi}$ of $\widehat{f}$, which is a parabolic complex Dulac series. By Theorem A, there exists a parabolic analytic linearization $\varphi$ of $f$ on the $f$-invariant subdomain $D:=\left(\mathcal{R}_{C}\right)_{R}$, given as the uniform limit on $D$ of the Koenigs sequence for $f$. Note that $D$ is a ( $f$-invariant) germ of $\mathcal{R}_{C}$. 
To prove that $\varphi$ is a complex Dulac germ, we prove that it admits $\widehat{\varphi}$ as its asymptotic expansion, uniformly on some standard quadratic subdomain $\mathcal{R}_{C^{\prime}}$ of $D$, as $\Re(\zeta) \rightarrow+\infty$.

Let $\varphi_{n}, n \in \mathbb{N}$, be the partial sums of the formal linearization $\widehat{\varphi}$ defined by (4.9), and let

$$
\psi_{n}(\zeta):=\varphi(\zeta)-\varphi_{n}(\zeta), \zeta \in D, n \in \mathbb{N} .
$$

Note that, by Theorem A (iii) and (4.9), for every $\delta>0$ such that $\beta_{1}-\delta>0$ it holds that

$$
\psi_{n}(\zeta)=\zeta+o(1)-\zeta-o\left(\mathrm{e}^{-\left(\beta_{1}-\delta\right) \zeta}\right)=o(1),
$$

uniformly on $D$ as $\Re(\zeta) \rightarrow+\infty$. Since $\varphi$ is an analytic linearization of $f$ on $D$, by (4.23) the following holds:

$$
\psi_{n}(f(\zeta))-\psi_{n}(\zeta)=-\varphi_{n}(f(\zeta))+\varphi_{n}(\zeta)+\beta, \text { for } \zeta \in D \text { and } n \in \mathbb{N} .
$$

By Lemma 4.3, for every $n \in \mathbb{N}$ there exists $\nu_{n}>0$ such that $\left(\varphi_{n} \circ f\right)(\zeta)-\varphi_{n}(\zeta)=$ $\beta+o\left(\mathrm{e}^{-\left(\beta_{n}+\nu_{n}\right) \zeta}\right)$, uniformly on $\mathcal{R}_{C}$ as $\Re(\zeta) \rightarrow+\infty$. Here, $\beta_{n}>0(n \in \mathbb{N})$ are the exponents in the complex Dulac series $\widehat{\varphi}$, as in (4.8). Now applying Lemma 4.4 to (4.25), for every $n \in \mathbb{N}$, the homological equation (4.25) admits a unique solution $\eta_{n}$ analytic on $D$, such that $\eta_{n}(\zeta)=O\left(\mathrm{e}^{-\left(\beta_{n}+\nu_{n}\right) \zeta}\right)$ uniformly on $D$, as $\Re(\zeta) \rightarrow+\infty$.

Since $\psi_{n}=o(1)$ by (4.24), it follows from Lemma $4.4(3)$ that $\psi_{n} \equiv \eta_{n}$ on $D$, $n \in \mathbb{N}$. Therefore,

$$
\psi_{n}(\zeta)=O\left(\mathrm{e}^{-\left(\beta_{n}+\nu_{n}\right) \zeta}\right), n \in \mathbb{N} .
$$

This implies, by (4.23), that $\widehat{\varphi}$ is the asymptotic expansion of the linearization $\varphi$ on $D$. Recall that $D$ is a germ of the standard quadratic domain $\mathcal{R}_{C}$, by Definition 2.1.

By Remark 2.2, there exists a standard quadratic subdomain $\mathcal{R}_{C^{\prime}}$, where $C^{\prime}>$ $R, C$, that is contained in $D$. Therefore, $\widehat{\varphi}$ is the Dulac asymptotic expansion of $\varphi$ also on the standard quadratic subdomain $\mathcal{R}_{C^{\prime}}$. Thus, $\varphi$ is a parabolic complex Dulac germ (its domain of definition contains standard quadratic domains).

Finally, the uniqueness of the linearization $\varphi$ follows from Theorem A (iv). The statement about real Dulac linearizations of real Dulac germs follows from Theorem A $(i i)$.

\section{REFERENCES}

[ABS19] I. Aniceto, G. Başar, and R. Schiappa, A primer on resurgent transseries and their asymptotics, Phys. Rep. 809 (2019), 1-135. 2

[CG93] L. Carleson and T. W. Gamelin, Complex dynamics, Universitext: Tracts in Mathematics, Springer-Verlag, New York, 1993. 2, 9

[DF03] D. J. Dewsnap and P. Fischer, Convergence of Koenigs' sequences, Real Anal. Exchange 28 (2002/03), no. 1, 111-120. 2, 4, 8, 9, 16

[DMM01] L. van den Dries, A. Macintyre, and D. Marker, Logarithmic-exponential series, Proceedings of the International Conference "Analyse \& Logique" (Mons, 1997), vol. 111, 2001, pp. 61-113. 22

[Éca92] J. Écalle, Introduction aux fonctions analysables et preuve constructive de la conjecture de Dulac, Actualités Mathématiques, Hermann, Paris, 1992. 2

[Il'84] Y. Il'yashenko, Limit cycles of polynomial vector fields with nondegenerate singular points on the real plane, Functional Anal. Appl. 18 (1984), no. 3, 199-209. 3, 7, 8

[Il'91] Finiteness theorems for limit cycles, Translations of Mathematical Monographs, vol. 94, American Mathematical Society, Providence, RI, 1991. 2, 4, 6

[IY08] Y. Ilyashenko and S. Yakovenko, Lectures on analytic differential equations, Graduate Studies in Mathematics, vol. 86, American Mathematical Society, Providence, RI, 2008. 5,6 
[Kne49] H. Kneser, Reelle analytische Lösungen der Gleichung $\varphi(\varphi(x))=e^{x}$ und verwandter Funktional-gleichungen, J. Reine Angew. Math. 187 (1949), 56-67. 2

[Koe84] G. Koenigs, Recherches sur les intégrales de certaines équations fonctionnelles, Ann. Sci. École Norm. Sup. (3) 1 (1884), 3-41. 2

[Lor98] F. Loray, Analyse des séries divergentes, Mathématiques pour le 2e cycle, vol. Quelques aspects des mathématiques actuelles, Ellipses, 1998. 9, 25

[Lor05] _ Pseudo-groupe d'une singularité de feuilletage holomorphe en dimension deux., Prépublication IRMAR, 2005. 3, 6, 7

[Mil06] J. Milnor, Dynamics in one complex variable, third ed., Annals of Mathematics Studies, vol. 160, Princeton University Press, Princeton, NJ, 2006. 2

[MR21] P. Mardešić and M. Resman, Analytic moduli for parabolic dulac germs, arXiv:1910.06129v2, to be published in Russian Mathematical Surveys, 2021. 3

[MRRŽ19] P. Mardešić, M. Resman, J.-P. Rolin, and V. Županović, The Fatou coordinate for parabolic Dulac germs, J. Differential Equations 266 (2019), no. 6, 3479-3513. 3, 4

[MRŽ12] P. Mardešić, M. Resman, and V. Županović, Multiplicity of fixed points and growth of ع-neighborhoods of orbits, J. Differential Equations 253 (2012), no. 8, 2493-2514. 3

[Nav11] A. Navas, Groups of circle diffeomorphisms, Chicago Lectures in Mathematics, University of Chicago Press, Chicago, IL, 2011. 2

[PRRS21] D. Peran, J.-P. Rolin, M. Resman, and T. Servi, Normal forms of hyperbolic logarithmic transseries, Preprint, March 2021. 1, 4, 8, 16, 17, 20, 21, 22

[R13] M. Resman, $\varepsilon$-neighborhoods of orbits and formal classification of parabolic diffeomorphisms, Discrete Contin. Dyn. Syst., 33, (2013), 3767-3790 3

[Rou98] R. Roussarie, Bifurcation of planar vector fields and Hilbert's sixteenth problem, Progress in Mathematics, vol. 164, Birkhäuser Verlag, Basel, 1998. 3, 7, 8

[Sch70] E. Schröder, Ueber iterirte Functionen, Math. Ann. 3 (1870), no. 2, 296-322. 1

[Ste57] S. Sternberg, Local $C^{n}$ transformations of the real line, Duke Math. J. 24 (1957), 97-102. 2

[Sze58] G. Szekeres, Regular iteration of real and complex functions, Acta Math. 100 (1958), 203-258. 2

[ZZ08] D. Žubrinić and V. Županović, Poincaré map in fractal analysis of spiral trajectories of planar vector fields, Bull. Belg. Math. Soc. Simon Stevin, 15 (2008), 947-960. 3

Addresses:

1: University of Split, Faculty of Science, Ruđera Boškovića 33, 21000 Split, Croatia, email: dino.peran@pmfst.hr

2: University of Zagreb, Faculty of Science, Department of Mathematics, Bijenička 30, 10000 Zagreb, Croatia, email: maja.resman@math.hr

3: Institut de Mathématiques de Bourgogne (UMR 5584 CNRS), Université de Bourgogne, Faculté des Sciences Mirande, 9 avenue Alain Savary, BP 47870, 21078 Dijon Cedex, France, email: jean-philippe.rolin@u-bourgogne.fr

4: Université de Paris and Sorbonne Université, CNRS, IMJ-PRG, F-75006 Paris, France, email: tamara.servi@imj-prg.fr 